\title{
Hidden symmetry and (super)conformal mechanics in a monopole background
}

\author{
Luis Inzunza, Mikhail S. Plyushchay and Andreas Wipf \\ Departamento de Física, Universidad de Santiago de Chile, \\ Casilla 30\%, Santiago, Chile \\ Theoretisch-Physikalisches Institut, Friedrich-Schiller-Universität Jena, \\ Max-Wien-Platz 1, 07743 Jena, Germany \\ E-mail: luis.inzunza@usach.cl, mikhail.plyushchay@usach.cl, \\ wipf@tpi.uni-jena.de
}

ABSTRACT: We study classical and quantum hidden symmetries of a particle with electric charge $e$ in the background of a Dirac monopole of magnetic charge $g$ subjected to an additional central potential $V(r)=U(r)+(e g)^{2} / 2 m r^{2}$ with $U(r)=\frac{1}{2} m \omega^{2} r^{2}$, similar to that in the one-dimensional conformal mechanics model of de Alfaro, Fubini and Furlan $(\mathrm{AFF})$. By means of a non-unitary conformal bridge transformation, we establish a relation of the quantum states and of all symmetries of the system with those of the system without harmonic trap, $U(r)=0$. Introducing spin degrees of freedom via a very special spin-orbit coupling, we construct the $\mathfrak{o s p}(2 \mid 2)$ superconformal extension of the system with unbroken $\mathcal{N}=2$ Poincaré supersymmetry and show that two different superconformal extensions of the one-dimensional AFF model with unbroken and spontaneously broken supersymmetry have a common origin. We also show a universal relationship between the dynamics of a Euclidean particle in an arbitrary central potential $U(r)$ and the dynamics of a charged particle in a monopole background subjected to the potential $V(r)$.

KEYWORDs: Conformal and W Symmetry, Extended Supersymmetry

ARXiv EPrint: 2002.04341 


\section{Contents}

1 Introduction $\quad 1$

2 Conformal mechanics in a monopole background 4

2.1 Classical dynamics 5

2.2 Conformal Newton-Hooke symmetry 8

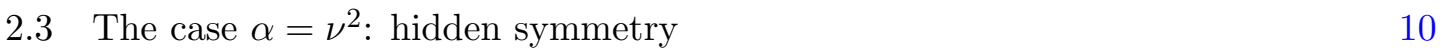

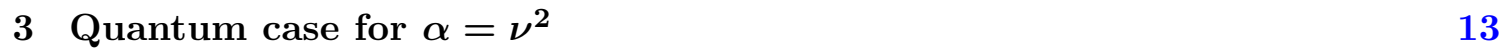

$\begin{array}{ll}3.1 \text { The algebraic approach } & 15\end{array}$

$\begin{array}{lll}3.2 & \text { The conformal bridge } & 17\end{array}$

4 A charge-monopole superconformal model $\quad 19$

4.1 Spin-orbit coupling model 20

4.2 The $\mathfrak{o s p}(2 \mid 2)$ superconformal extension $\quad 22$

$\begin{array}{lll}4.3 & \text { Dimensional reduction } & 26\end{array}$

5 Discussion and outlook $\quad 30$

A The monopole harmonics $\quad 34$

B The derivation of $A_{n, j, m}$ and $B_{n, j, m}$

$\begin{array}{ll}\text { C Generalized Laguerre polynomials } & 37\end{array}$

$\begin{array}{ll}\text { D Commutators }[\mathcal{R}, \mathcal{Q}] & \text { and }\left[\mathcal{R}, \mathcal{W}^{\dagger}\right] \\ \end{array}$

\section{Introduction}

Hidden symmetries are associated with peculiar classical and quantum properties of a system [1]. They are generated by higher order in canonical momenta integrals of motion. When a generator of a hidden symmetry does not depend explicitly on time, it transforms solutions of a system into solutions having the same energy. Otherwise, a symmetry generator is the integral of motion which explicitly depends on time and relates solutions of different energies. Hidden symmetries appear in a broad spectrum of the systems, including the Kepler-Coulomb problem, anisotropic harmonic oscillator with commensurable frequencies, Higgs oscillator [2] and the Klein-Gordon equation in Anti-de Sitter spacetime [3, 4], integrable nonlinear wave equations [5], Calogero model [6], Kerr-Newman, or more general Kerr-NUT-(A)dS black hole solutions of the Einstein-Maxwell equations [7]. 
They also reveal themselves nontrivially in supersymmetric extensions of such systems, both in non-exotic $[8,9]$ and exotic $[10,11]$.

One of the most known examples of hidden symmetries corresponds to the case of the three-dimensional isotropic harmonic oscillator, where the closed character of the trajectories is encoded in the Fradkin's tensor integral [12], which is analogous to the LaplaceRunge-Lentz vector in the Kepler-Coulomb problem. These tensor and vector integrals together with angular momentum vectors of the systems define the elliptic form of particle's trajectories and their spatial orientation, and at the quantum level the presence of these integrals explains the origin of the so called "accidental" spectral degeneracy $[13,14]$.

Another example is provided by reflectionless and finite-gap quantum systems intimately related to the Korteweg-de Vries and modified Korteweg-de Vries equations, in which the higher-derivative Lax-Novikov integrals separate the left- and right-moving Bloch states and detect all the bound states and the states at the edges of the continuos parts (bands) of their spectra by annihilating them. Those integrals give rise to appearance of exotic nonlinear supersymmetric structures in super-extended versions of such systems $[10,11,15]$.

Explicitly depending on time, dynamical higher order in momenta integrals of motion appear in rational extensions of one-dimensional conformally invariant systems where they detect and encode the fine, finite-gap type spectral structure [16-20]. In these systems as well as in general case the higher derivative in momenta generators of hidden symmetries give rise to non-linear generalizations of Lie algebras and superalgebras [21].

Yet another example of the hidden symmetries corresponds to a non-standard extension of the fermion-monopole supersymmmetry [22, 23], the existence of which can be related to the Killing-Yano tensor admitted by the flat background of the monopole [24]. In this sense its origin is similar to the origin of the exotic "SUSY in the sky" of Gibbons, Rietdijk and van Holten [25-27], in which additional supercharges are related to generators of hidden symmetries as it happens in the case of diverse black-hole solutions of the Einstein-Maxwell equations in $(3+1)$ and higher dimensions [7]. Alternatively, exotic supersymmetry of the fermion-monopole system finds a simple explanation in special properties of the dynamics of a spin- $1 / 2$ charged particle in monopole background [28].

The flat background of the monopole is revealed in the dynamics of a scalar particle with electric charge $e$ which in its field realizes a force-free, geodesic motion on a surface of a dynamical cone defined by the charge-monopole coupling parameter $\nu=e g$, where $g$ is the monopole's magnetic charge [29-31]. One can consider a more general case of the charged particle in the monopole background subjected to the action of an additional central potential of the form

$$
V(r)=\frac{\alpha}{2 m r^{2}}+U(r),
$$

where the first term is conformally invariant and $U(r)$ is a smooth function of $r=\sqrt{r^{2}}$. Earlier results [32] of two of us show that in the case $U(r)=0$ and particular value of the coupling $\alpha=\nu^{2}$, the projection of the particle's trajectory to the plane ortogononal to the total angular momentum vector of the system corresponds to the one-dimensional free motion along a straight line defined by a certain analog of the Laplace-Runge-Lenz vector 
which for the free particle is $\boldsymbol{p} \times \boldsymbol{L}$. It looks like the particle in the field of the monopole "remembers" the integrals of motion of the system with switched off charge-monopole coupling $(\nu \rightarrow 0)$. Supersymmetric extension of such a system is described by the Pauli Hamiltonian of a spin-1/2 particle in background of a self-dual or anti-self-dual dyon, which is characterized by a nonlinear, quadratically extended Lie superalgebra $D(2,1 ; 1 / 2)$ [32] being a particular case of the exceptional superagebra $D(2,1 ; \alpha)[9]$.

From another perspective, in the absence of the monopole background $(g=0)$, two cases of the systems described by potential (1.1) with $U(r)=0$ and $U(r)=\frac{1}{2} m \omega^{2} r^{2}$ are intimately related to each other and represent two forms of dynamics in the sense of Dirac [33] corresponding to conformal symmetry. The integrals of the system with $U(r)=0$ can be obtained by taking some linear combinations of integrals of another system and applying to them a limit $\omega \rightarrow 0$. Or, in both directions the systems and their integrals can be related at classical and quantum levels by a non-unitary mapping corresponding to the conformal bridge transformation considered recently by us in ref. [34].

Based on the described relations and peculiarities, one can conjecture that in the presence of the monopole and confining harmonic term $U=\frac{1}{2} m \omega^{2} r^{2}$ in potential (1.1) something particular (related to hidden and conformal symmetries) should happen in the special case $\alpha=\nu^{2}$. One could expect similar peculiar properties to be seen also in a superextended version of such a system. If so, it would be an interesting result from the point of view of three-dimensional (or more generally higher-dimensional) supersymmetric quantum mechanics, because contrary to the one-dimensional case [35-38], there is no canonical way to obtain such systems. Although there are particular and elegant constructions, see for example $[8,9,32,39-43]$, it is in general a non-trivial task to produce such theories. In the present context, a possible approach would be to look for a general $(3+0)$ dimensional Dirac type operator as a supercharge (square root) of a Klein-Gordon type "super-Hamiltonian".

This work is devoted to the investigation of the conjectures specified in the previous paragraph, and in conclusion of this section we describe the organization of the paper and briefly summarize its results.

In section 2 we investigate the classical theory of a charged scalar particle in a monopole background subjected to the action of additional scalar potential of the form (1.1) with a harmonic trap $U(r)=\frac{1}{2} m \omega^{2} r^{2}$, which has a nature similar to the potential in onedimensional conformal mechanics model of de Alfaro, Fubini and Furlan (AFF) [44] described by the Hamiltonian ${ }^{1}$

$$
H_{\mathrm{AFF}}=\frac{p^{2}}{2 m}+\frac{m \omega^{2} q^{2}}{2}+\frac{\ell(\ell+1)}{2 m q^{2}} .
$$

We solve the equations of motion and find that the trajectories are closed for an arbitrary choice of initial conditions only in the special case when $\alpha=\nu^{2}$. We show that in this special case the dynamics of the radial variable is governed by conformal Newton-Hooke symmetry of the system and compare it with the dynamics of the system with $U(r)=0$

\footnotetext{
${ }^{1}$ This model and its supersymmetric extensions [45, 46] play important role, in particular, in black hole physics [47-50], AdS/CFT correspondence [51, 52], cosmology [53, 54], and holographic QCD [55].
} 
studied earlier in details in ref. [32]. It turns out that in the special case $\alpha=\nu^{2}$ the full dynamics - including its angular part - is controlled by a hidden symmetry described by the integrals of motion of order four in momenta variables. These integrals define the orientation of the trajectory projected to the plane orthogonal to the conserved total angular momentum vector. In spite of the fourth order in momenta nature of generators of the hidden symmetry, in contrast with the second order generators for the isotropic harmonic oscillator, they reveal a structure somehow similar to the Fradkin's tensor in the latter system. Section 3 is devoted to the quantum theory of the system with $\alpha=\nu^{2}$, where, in particular, with the help of the hidden symmetry we identify the full set of its ladder operators. Using the results of our previous paper [34], we also construct the conformal bridge transformation which relates the quantum spectrum of our system with that of the model without confining harmonic term as has been studied in [32]. Particularly, we show that coherent states for the present system are generated by the conformal bridge transformation from non-normalizable energy eigenstates of the system with $U(r)=0$, while all its energy eigenstates are produced from Jordan states of zero energy of the latter system. This transformation also establishes a relation between symmetries of both systems, including generators of their hidden symmetries. In section 4 we consider a supersymmetric generalization of the quantum system by introducing a very special spin-orbit coupling, that allows us to obtain the $\mathfrak{o s p}(2 \mid 2)$ superconformal extension of the system with unbroken $\mathcal{N}=2$ Poincaré supersymmetry. We also demonstrate that two different superconformal extensions of the one-dimensional AFF model with unbroken and spontaneously broken phases of $\mathcal{N}=2$ Poincaré supersymmetry have a common origin in the three-dimensional $\mathfrak{o s p}(2 \mid 2)$ superconformal symmetry of the spin-1/2 particle in a monopole background. When switching off the monopole background by setting $g=0$, the non-relativistic limit of the Dirac oscillator considered in refs. [56-60] is recovered, and the $\mathfrak{o s p}(2 \mid 2)$ superconformal symmetry remains intact. On the other hand, when switching off the harmonic trap by taking $\omega=0$, the superconformal Hamiltonian of our extended system takes the form of the Pauli type Hamiltonian for a charged spin- $1 / 2$ particle in a field of the self-dual dyon studied in [32]. The discussion of our results and an outlook are presented in section 5, where we also generalize the observation of section 2 by showing a universal relationship between the three-dimensional dynamics of a Euclidean particle in an arbitrary central potential $U(r)$ and the dynamics of a charged particle in a monopole background subjected to the action of the central potential $U(r)+\nu^{2} / 2 m r^{2}$. Several technical details are moved to four appendices.

\section{Conformal mechanics in a monopole background}

In this section we study the dynamics of a charged particle in background of a magnetic monopole in the presence of an additional central potential which is a three-dimensional analog of that in the AFF conformal mechanics model [44]. The system we investigate is given by the Hamiltonian

$$
H=\frac{\pi^{2}}{2 m}+\frac{m \omega^{2} r^{2}}{2}+\frac{\alpha}{2 m r^{2}}
$$


where $\omega>0, \boldsymbol{\pi}=\boldsymbol{p}-e \boldsymbol{A}, \boldsymbol{A}$ is a U(1) gauge potential of a Dirac magnetic monopole at the origin with charge $g, \nabla \times \boldsymbol{A}=\boldsymbol{B}=g \boldsymbol{r} / r^{3}$, and the coupling $\alpha$ should be chosen appropriately to prevent a fall to the center, see below. We solve the Hamiltonian equations, study the conformal Newton-Hooke symmetry of the system, and investigate a hidden symmetry which appears in a special case $\alpha=\nu^{2}, \nu=e g$. We follow here the line of reasoning used in [32] to identify the hidden symmetry and characterize the particle's trajectories.

\subsection{Classical dynamics}

The particle's coordinates and kinetic momenta obey the Poisson brackets relations

$$
\left\{r_{i}, \pi_{j}\right\}=\delta_{i j}, \quad\left\{r_{i}, r_{j}\right\}=0, \quad\left\{\pi_{i}, \pi_{j}\right\}=e \epsilon_{i j k} B_{k},
$$

which give rise to the equations of motion

$$
\dot{\boldsymbol{r}}=\frac{1}{m} \boldsymbol{\pi}, \quad \dot{\boldsymbol{\pi}}=\frac{1}{m r^{3}}(\alpha \boldsymbol{n}-\nu \boldsymbol{r} \times \boldsymbol{\pi})-m \omega^{2} \boldsymbol{r},
$$

where $\boldsymbol{n}=\boldsymbol{r} / \boldsymbol{r}$. From (2.3) we derive the equations

$$
\frac{d r}{d t}=\frac{1}{m} \pi_{r}, \quad \dot{n}=\frac{1}{m r^{2}} \boldsymbol{J} \times \boldsymbol{n},
$$

where we denote $\pi_{r}=\boldsymbol{n} \cdot \boldsymbol{\pi}$, and

$$
J=r \times \pi-\nu n
$$

is the conserved Poincaré vector identified as the angular momentum of the system,

$$
\left\{J_{i}, J_{j}\right\}=\epsilon_{i j k} J_{k}, \quad\left\{J_{i}, r_{j}\right\}=\epsilon_{i j k} r_{k}, \quad\left\{J_{i}, \pi_{j}\right\}=\epsilon_{i j k} \pi_{k} .
$$

From (2.5) it follows that $\boldsymbol{J} \cdot \boldsymbol{n}=-\nu$ and $\boldsymbol{J}^{2} \geq \nu^{2}$, i.e. a trajectory of the particle lies on the surface of a cone with symmetry axis given by the angular momentum vector $\boldsymbol{J}$ and cone's angle

$$
\theta=\arccos (-\nu / J), \quad J=\sqrt{J^{2}} .
$$

In the limit case $J^{2}=\nu^{2}$ the cone degenerates into a half-line. If $\nu<0$, then $\theta=0$ and the particle moves on a half-line directed along the angular momentum $\boldsymbol{J}$, whereas $\theta=\pi$ if $\nu>0$ and the particle moves on a half-line opposite to the direction of the vector $\boldsymbol{J}$.

By means of eq. (2.5), the Hamiltonian can be presented in the form

$$
H=\frac{\pi_{r}^{2}}{2 m}+\frac{\mathscr{L}^{2}}{2 m r^{2}}+\frac{m \omega^{2} r^{2}}{2}, \quad \mathscr{L}^{2}:=\boldsymbol{J}^{2}-\nu^{2}+\alpha,
$$

which shows that the radial dynamical variables $r$ and $\pi_{r},\left\{r, \pi_{r}\right\}=1$, behave like $q$ and $p$ in the one dimensional AFF model (1.2). From (2.8) it follows that there is no fall to the center if $\mathscr{L}^{2}>0$, i.e. $\alpha>0$, that we will assume from now on. Eq. (2.8) also implies that the possible values of the angular momentum $J$ and energy obey the relation

$$
\frac{\mathscr{L} \omega}{H}:=\lambda \leq 1
$$




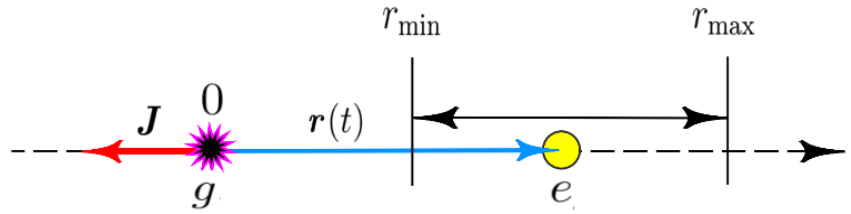

Figure 1. Illustration for the case $J^{2}=\nu^{2}$ with $\nu=e g>0$. The monopole with charge $g$ is at the origin of the coordinate system, and the vectors $\boldsymbol{J}$ and $\boldsymbol{r}$ are oriented in opposite directions. The position of the particle of charge $e$ oscillates between $r_{\min }$ and $r_{\max }$. For $\nu<0$ the vector $\boldsymbol{J}$ is oriented in the same direction as $r$.

Let $r_{0}=r\left(t_{0}\right)$ corresponds to a turning point, $\dot{r}\left(t_{0}\right)=0$. Then according to relation $\pi_{r}=m \dot{r}$ and (2.8), $r_{0}^{2}$ is defined by the equation

$$
r_{0}^{4}-\frac{2 H}{m \omega^{2}} r_{0}^{2}+\frac{\mathscr{L}^{2}}{m^{2} \omega^{2}}=0
$$

Its two solutions

$$
r_{ \pm}^{2}=\frac{H}{m \omega^{2}}(1 \pm \rho), \quad 0 \leq \rho=\sqrt{1-\lambda^{2}}<1
$$

satisfy the relation

$$
r_{+} r_{-}=\frac{\mathscr{L}}{m \omega} .
$$

If, for simplicity, we choose the initial moment of time $t_{0}=0$ such that $r(0)=r_{-}=r_{\min }$, integration of the first equation in (2.4) with taking into account eq. (2.8) yields

$$
r^{2}(t)=\frac{H}{m \omega^{2}}(1-\rho \cos (2 \omega t)) .
$$

So, $r(t)$ oscillates between $r_{\min }=r_{-}$and $r_{\max }=r_{+}$with a period $\pi / \omega$. The particular case with $H=\mathscr{L} \omega$ corresponds to a circular motion for which $r(t)=r_{+}=r_{-}=(\mathscr{L} / m \omega)^{1 / 2}$. If $J^{2}=\nu^{2}$, the particle realizes one-dimensional oscillations (2.13) between $r_{\min }$ and $r_{\max }$ lying on the half-line specified below eq. (2.7), see figure 1

To solve the vector equation in (2.4) in the case $J^{2}>\nu^{2}$, which we will assume in what follows, we decompose $\boldsymbol{n}$ into the component parallel to the angular momentum and the orthogonal component,

$$
\boldsymbol{n}(t)=\boldsymbol{n}_{\|}+\boldsymbol{n}_{\perp}(t)=-\nu \frac{\hat{\boldsymbol{J}}}{J}+\boldsymbol{n}_{\perp}(t), \quad \boldsymbol{J} \cdot \boldsymbol{n}_{\perp}(t)=0,
$$

where $\hat{\boldsymbol{J}}$ is the unit vector in the direction of $\boldsymbol{J}$. Since the parallel component $\boldsymbol{n}_{\|}$is constant, we conclude that the orthogonal component $\boldsymbol{n}_{\perp}(t)$ has constant length and thus describes a circle in the plane orthogonal to $\boldsymbol{J}$,

$$
\boldsymbol{n}_{\perp}(t)=\boldsymbol{n}_{\perp}(0) \cos \varphi(t)+\hat{\boldsymbol{J}} \times \boldsymbol{n}_{\perp}(0) \sin \varphi(t) .
$$

Using the second equation in (2.4) we then obtain

$$
\dot{\varphi}=\frac{J}{m r^{2}},
$$


that yields the time-dependence of the angular variable. ${ }^{2}$ Integrating this equation with using (2.13) and assuming $\varphi(0)=0$, we obtain

$$
\varphi(t)=\frac{J}{\mathscr{L}} \arctan \left(\frac{r_{\max }}{r_{\min }} \tan (\omega t)\right) .
$$

It is convenient to parametrize the orbits by expressing $\xi=1 / r^{2}$ as a function of $\varphi$. We have $d \xi / d \varphi=-2 r^{-3} \dot{r} / \dot{\varphi}$, and so,

$$
\frac{d \xi}{d \varphi}=-\frac{2}{J} \sqrt{2 m H \xi-m^{2} \omega^{2}-\mathscr{L}^{2} \xi^{2}} .
$$

Integration of this equation yields

$$
\xi(\varphi)=\frac{1}{r^{2}(\varphi)}=\frac{m H}{\mathscr{L}^{2}}\left[1+\rho \cos \left(\frac{2 \mathscr{L}}{J} \varphi\right)\right]
$$

which corresponds to $r(\varphi=0)=r_{\min }$ and the angular period $\pi J / \mathscr{L}$. The condition for a periodic trajectory is

$$
\frac{2 \mathscr{L}}{J} 2 \pi l_{r}=2 \pi l_{a} \quad \Longleftrightarrow \quad \frac{2 \mathscr{L}}{J}=\frac{l_{a}}{l_{r}}, \quad l_{r}, l_{a}=1,2, \ldots
$$

From the definition of $\mathscr{L}$ in (2.8) we find that the trajectories are closed for arbitrary values of $J$ if and only if $\alpha=\nu^{2}$. If $\alpha \neq \nu^{2}$, the trajectory will be closed only for special values of the angular momentum given by the condition

$$
\alpha=\nu^{2}+\left(\frac{1}{4} \frac{l_{a}^{2}}{l_{r}^{2}}-1\right) J^{2},
$$

and in this case eq. (2.9) takes the form $\frac{l_{a}}{l_{r}} \leq \frac{2 H}{\omega J}$.

Figure 2 illustrates several particular orbits lying on the corresponding conical surface in a general case $\alpha \neq \nu^{2}$ and in the special case $\alpha=\nu^{2}$. Trajectories $r(\varphi)$ are shown there for fixed values of $H, \boldsymbol{J}$ and $\nu$, but for different values of $\alpha$. Below we shall see that when $\alpha=\nu^{2}$, the projection to the plane orthogonal to $\boldsymbol{J}$ of the trajectory shown on the last plot is an ellipse centered at the origin of the coordinate system similarly to the case of the three-dimensional isotropic harmonic oscillator. This corresponds to a fundamental universal property of the magnetic monopole background which we discuss in the last section. Since the center of the projected elliptical trajectory is in the center of an ellipse, the angular period $P_{a}$ is twice the radial period $P_{r}, P_{a} / P_{r}=2$, similarly to the isotropic harmonic oscillator. This is different from the picture of the finite orbits in Kepler problem where the force center is in one of the foci, and as a result $P_{a}=P_{r}$. This similarity with the isotropic oscillator and contrast to the Kepler problem are also reflected in the spectra of the systems at the quantum level.

\footnotetext{
${ }^{2}$ Eqs. (2.15) and (2.16) imply a rotation of $\boldsymbol{n}_{\perp}$ in the positive, clockwise direction looking on it from the direction of the vector $\boldsymbol{J}$. If $\boldsymbol{J}$ is oriented along $\boldsymbol{e}_{z}$, and $\nu<0,0<\theta<\pi / 2$ in (2.7), the particle's trajectory lies on the upper sheet of the cone and $\boldsymbol{n}_{\perp}$ rotates in a clockwise direction in the horizontal plane. If $\boldsymbol{J}$ is oriented along $-\boldsymbol{e}_{z}$, and $\nu>0, \pi / 2<\theta<\pi$, then the trajectory lies again on the upper sheet of the cone, but the vector $\boldsymbol{n}_{\perp}$ rotates anti-clockwise in the $(x, y)$ plane if to look at it from $\boldsymbol{e}_{z}$.
} 

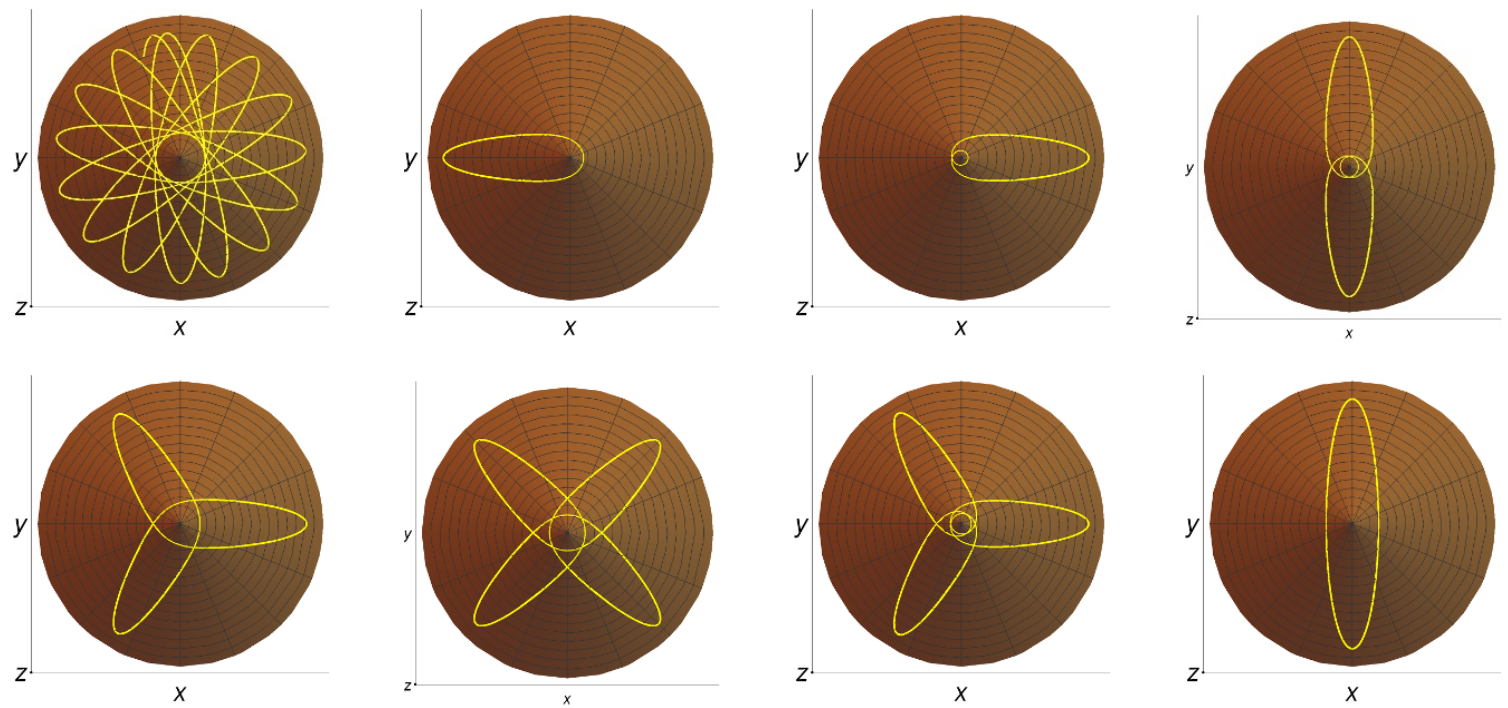

Figure 2. The depicted trajectories correspond to the vector $\boldsymbol{J}$ oriented along $\boldsymbol{e}_{z}$. The first figure in the top row represents the generic case with non-closed trajectory. The other figures are examples of closed trajectories with parameters satisfying the relation $(2.21)$, with quotients $l_{a} / l_{r}=$ $\{1,1 / 2,2 / 3,3 / 2,4 / 3,3 / 4,2\}$ are sequentially shown. The last relation $l_{a} / l_{r}=2$ corresponds to the special case $\alpha=\nu^{2}$.

\subsection{Conformal Newton-Hooke symmetry}

In this subsection we derive the conformal Newton-Hooke symmetry [61-64] for the system (2.1) and compare it with the conformal symmetry of the model with $\omega=0$ studied in $[32]$.

Using the AFF form of the Hamiltonian (2.8), one can show that the complex quantity

$$
\mathcal{C}=e^{2 i \omega t}\left(\frac{\pi_{r}^{2}}{2 m}+\frac{\mathscr{L}^{2}}{2 m r^{2}}-\frac{m \omega^{2} r^{2}}{2}-i \omega r \pi_{r}\right)=e^{2 i \omega t}\left(H-m \omega^{2} r^{2}-i \omega r \pi_{r}\right),
$$

and its complex conjugate $\mathcal{C}^{*}$ are explicitly depending on time integrals of motion which together with $H$ generate the $\mathfrak{s l}(2, \mathbb{R})$ algebra

$$
\{H, \mathcal{C}\}=2 i \omega \mathcal{C}, \quad\left\{H, \mathcal{C}^{*}\right\}=-2 i \omega \mathcal{C}^{*}, \quad\left\{\mathcal{C}, \mathcal{C}^{*}\right\}=-4 i \omega H
$$

In terms of $\mathcal{C}$ and $\mathcal{C}^{*}$, the generators of the Newton-Hooke symmetry are given by

$$
D=\frac{i}{4 \omega}\left(\mathcal{C}-\mathcal{C}^{*}\right), \quad K=\frac{1}{4 \omega^{2}}\left(2 H-\mathcal{C}-\mathcal{C}^{*}\right),
$$

and together with $H$ they satisfy the algebra

$$
\{D, H\}=H-2 \omega^{2} K, \quad\{D, K\}=-K, \quad\{K, H\}=2 D,
$$

whose Casimir invariant is

$$
\mathscr{F}=D^{2}+\omega^{2} K^{2}-K H=-\frac{1}{4} \mathscr{L}^{2} .
$$



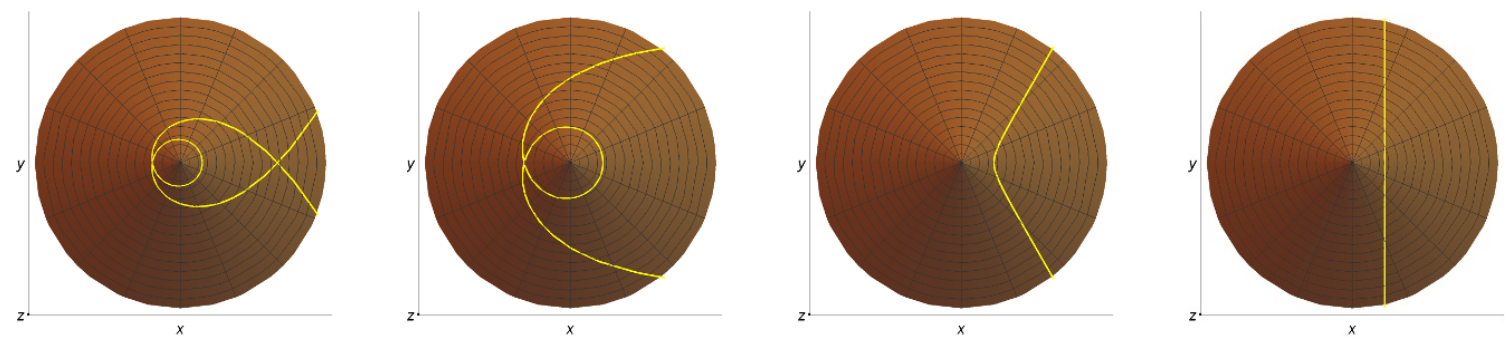

Figure 3. Each plot represents a trajectory for a specific value of $\alpha$ chosen according to (2.21) with the vector $\boldsymbol{J}$ oriented along $\boldsymbol{e}_{z}$. From left to right the cases $l_{a} / l_{r}=\{3 / 2,1 / 2,3,2\}$ are shown, where the last plot corresponds to the special case $\alpha=\nu^{2}$.

In terms of these generators, the function $r^{2}(t)$ is presented in the form

$$
r^{2}(t)=\frac{2}{m \omega^{2}}\left(\omega D \sin (2 \omega t)+\omega^{2} K \cos (2 \omega t)+H \sin ^{2}(\omega t)\right) .
$$

The values of the dynamical integrals $D$ and $K$ depend on the choice of initial conditions for $r$ and $\dot{r}$, and as follows from (2.22) and (2.24), our choice $r(0)=r_{\min }, \dot{r}(0)=0$ corresponds to $D=0$ and $K=\frac{1}{2} m r_{\min }^{2}$. For these values of $D$ and $K,(2.27)$ takes the form (2.13). On the other hand, since $\boldsymbol{J} \cdot \boldsymbol{r}(t)=-\nu \boldsymbol{r}(t)$, for a general choice of initial conditions eq. (2.27) shows that the dynamics of the projection of $\boldsymbol{r}(t)$ on the direction of the conserved angular momentum is controlled by the conformal Newton-Hooke symmetry of the system. According to (2.27), the oscillation period of $r(t)$ is $\pi / \omega$, and taking into account the value of the Casimir invariant, one can check that in the general case eq. (2.27) also implies that $r(t)$ oscillates between the values $r_{\min }$ and $r_{\max }$ given by eq. (2.11).

To conclude this part of the analysis, we comment on the limit $\omega \rightarrow 0$. In this case the generators $H, D$ and $K$ take the form

$$
H_{0}=\frac{\pi_{r}^{2}}{2 m}+\frac{\mathscr{L}^{2}}{2 m r^{2}}, \quad D_{0}=\frac{1}{2} r \pi_{r}-H_{0} t, \quad K_{0}=\frac{m r^{2}}{2}-D t-H_{0} t^{2},
$$

and satisfy the conformal algebra

$$
\left\{D_{0}, H_{0}\right\}=H_{0}, \quad\left\{D_{0}, K_{0}\right\}=-K_{0}, \quad\left\{K_{0}, H_{0}\right\}=2 D_{0}
$$

The case $\alpha=0$ of the system $H_{0}$ corresponds to a geodesic motion on the dynamical cone [30,31]. The special case of $\alpha=\nu^{2}$, on the other hand, was studied in [32]. It was shown there that the trajectory of the particle, projected to the plane orthogonal to $\boldsymbol{J}$, is a straight line along which the projected particle's motion takes place with constant velocity. Consistently with these peculiar properties, in the special case $\alpha=\nu^{2}$ the system with $H_{0}$ possesses a hidden symmetry described by the integral of motion $\boldsymbol{V}=\boldsymbol{\pi} \times \boldsymbol{J}$ being a sort of Laplace-Runge-Lenz vector, in the plane orthogonal to which and parallel to $\boldsymbol{J}$ the particle's trajectory lies [32]. In figure 3 some plots of the trajectories are shown for the system (2.28). 


\subsection{The case $\alpha=\nu^{2}$ : hidden symmetry}

In the case $\alpha=\nu^{2}$ the particle described by the Hamiltonian (2.1) admits additional integrals of motion responsible for the closed nature of the trajectories for arbitrary choice of initial conditions. The integrals are derived by an algebraic approach as in Fradkin's construction for the isotropic three-dimensional harmonic oscillator [12].

Let us first consider arbitrary values of $\alpha$ assuming, as we indicated above, $J^{2}>\nu^{2}$. Our first step is to introduce the vector quantities

$$
\begin{aligned}
& \boldsymbol{I}_{1}=\boldsymbol{\pi} \times \boldsymbol{J} \cos (\omega t)+\omega m \boldsymbol{r} \times \boldsymbol{J} \sin (\omega t), \\
& \boldsymbol{I}_{2}=\boldsymbol{\pi} \times \boldsymbol{J} \sin (\omega t)-\omega m \boldsymbol{r} \times \boldsymbol{J} \cos (\omega t) .
\end{aligned}
$$

The time-dependence of these vectors in the plane orthogonal to $\boldsymbol{J}$ follows from the timedependence of $\boldsymbol{\pi}$ and $\boldsymbol{r}$ in (2.3),

$$
\frac{d \boldsymbol{I}_{1}}{d t}=\frac{1}{m r^{3}}\left(\alpha-\nu^{2}\right) \boldsymbol{n} \times \boldsymbol{J} \cos (\omega t), \quad \frac{d \boldsymbol{I}_{2}}{d t}=\frac{1}{m r^{3}}\left(\alpha-\nu^{2}\right) \boldsymbol{n} \times \boldsymbol{J} \sin (\omega t) .
$$

Then

$$
\boldsymbol{I}_{1} \cdot \boldsymbol{I}_{2}=\frac{1}{2}\left(\nu^{2}-\alpha\right)\left(J^{2}-\nu^{2}\right) \frac{\sin (2 \omega t)}{2 r^{2}},
$$

where we have taken into account our choice of the initial condition $\pi_{r}(0)=0$. The initial values of these vectors are

$$
\boldsymbol{I}_{1}(0)=\frac{J^{2}}{r_{\min }} \boldsymbol{n}_{\perp}(0), \quad \boldsymbol{I}_{2}(0)=m \omega r_{\min } \boldsymbol{J} \times \boldsymbol{n}_{\perp}(0) .
$$

Thus, $\boldsymbol{I}_{1}$ and $\boldsymbol{I}_{2}$ are orthogonal to each other at $t=0$, but in general case of $\alpha \neq \nu^{2}$ their scalar product is not zero and changes periodically with period $\pi / \omega$.

For the particular choice $\alpha=\nu^{2}$, the vectors $\boldsymbol{I}_{1}$ and $\boldsymbol{I}_{2}$ are orthogonal vector integrals of motion of order 2 in the kinetic momenta, and so, they correspond to the "hidden symmetries" [1] of the system. They are, however, dynamical, explicitly time-dependent integrals of motion (similarly to generators of the conformal Newton-Hook symmetry $D$ and $K), \frac{d}{d t} \boldsymbol{I}_{1,2}=\frac{\partial \boldsymbol{I}_{1,2}}{\partial t}+\left\{\boldsymbol{I}_{1,2}, H\right\}=0$. Their lengths are also dynamical integrals whose values, again in the sense of a total time derivative, take constant values

$$
\left|\boldsymbol{I}_{1}\right|=m \omega \sqrt{J^{2}-\nu^{2}} r_{\max }, \quad\left|\boldsymbol{I}_{2}\right|=m \omega \sqrt{J^{2}-\nu^{2}} r_{\min },
$$

where we have taken into account eqs. (2.34) and (2.12). The sum of their squares, however, is a true integral of motion whose value is a function of $H$ and $J$,

$$
\boldsymbol{I}_{1}^{2}+\boldsymbol{I}_{2}^{2}=2 m H\left(J^{2}-\nu^{2}\right)
$$

These vectors point in the direction of the semi-axes of the elliptic trajectory in the plane orthogonal to $\boldsymbol{J}$. The lengths of semi-major and semi-minor axes correspond to those of the vectors $r \boldsymbol{n}_{\perp}(0)$ and $r \hat{\boldsymbol{J}} \times \boldsymbol{n}_{\perp}(0)$, and are equal to $r_{\max } \sqrt{1-\nu^{2} / J^{2}}$, and $r_{\min } \sqrt{1-\nu^{2} / J^{2}}$. We note that in general case $\alpha \neq \nu^{2}$ the periodic change of the scalar product of $\boldsymbol{I}_{1}$ and $\boldsymbol{I}_{2}$ implies a precession of the orbit, see figure 2 . 
Let us now investigate in more detail the most interesting case $\alpha=\nu^{2}$ given by the Hamiltonian

$$
H=\frac{\pi^{2}}{2 m}+\frac{m \omega^{2}}{2} r^{2}+\frac{\nu^{2}}{2 m r^{2}} .
$$

To express the general solution in terms of the conserved $\boldsymbol{J}$ and dynamical integrals $\boldsymbol{I}_{1}$ and $\boldsymbol{I}_{2}$ in (2.30) and (2.31), which for $\alpha=\nu^{2}$ become true integrals of motion, we note that

$$
\boldsymbol{r}(t) \times \boldsymbol{J}=\frac{1}{m \omega}\left(\boldsymbol{I}_{1} \sin (\omega t)-\boldsymbol{I}_{2} \cos (\omega t)\right), \quad \boldsymbol{\pi}(t) \times \boldsymbol{J}=\boldsymbol{I}_{1} \cos (\omega t)+\boldsymbol{I}_{2} \sin (\omega t) .
$$

By means of the relations

$$
\boldsymbol{J} \times(\boldsymbol{r}(t) \times \boldsymbol{J})=J^{2} \boldsymbol{r}(t)+\nu r(t) \boldsymbol{J}, \quad|\boldsymbol{r} \times \boldsymbol{J}|^{2}=\left(J^{2}-\nu^{2}\right) r^{2}(t),
$$

we can express the position $\boldsymbol{r}(t)$ of the particle as follows,

$$
\boldsymbol{r}(t)=\frac{1}{m \omega J^{2}}\left(\boldsymbol{J} \times \boldsymbol{I}_{1} \sin \omega t-\boldsymbol{J} \times \boldsymbol{I}_{2} \cos \omega t-\nu \frac{\sqrt{I_{1}^{2} \sin ^{2} \omega t+I_{2}^{2} \cos ^{2} \omega t}}{\sqrt{J^{2}-\nu^{2}}} \boldsymbol{J}\right),
$$

with $\boldsymbol{I}_{1}=\boldsymbol{I}_{1}(0)$ and $\boldsymbol{I}_{2}=\boldsymbol{I}_{2}(0)$. This yields us $\boldsymbol{r}(t)$ and kinetic momentum $\boldsymbol{\pi}=m \dot{\boldsymbol{r}}$ at any given time presented in terms of the angular momentum and dynamical vector integrals.

Alternatively, one can follow a more algebraic approach to extract information on the trajectories without explicitly solving the equations of motion. It is well known from the seminal paper of Fradkin [12] that for the three-dimensional isotropic harmonic oscillator all symmetries of the trajectories are encoded in a tensor integral of motion. In the remainder of this subsection we construct an analogous tensor for the system at hand to find the trajectories by a linear algebra techniques. We begin with the tensor integrals

$$
T^{i j}=T^{(i j)}+T^{[i j]}, \quad T^{(i j)}=\frac{1}{2}\left(I_{1}^{i} I_{1}^{j}+I_{2}^{i} I_{2}^{j}\right), \quad T^{[i j]}=\frac{1}{2}\left(I_{1}^{i} I_{2}^{j}-I_{1}^{j} I_{2}^{i}\right) .
$$

They, unlike the vectors $\boldsymbol{I}_{1}$ and $\boldsymbol{I}_{2}$, but like the quadratic expression (2.36) are the true, not depending explicitly on time integrals of motion, $\frac{d}{d t} T^{i j}=\left\{T^{i j}, H\right\}=0$, whose explicit form in phase space variables is

$$
2 T^{i j}=(\boldsymbol{\pi} \times \boldsymbol{J})^{i}(\boldsymbol{\pi} \times \boldsymbol{J})^{j}+m^{2} \omega^{2}(\boldsymbol{r} \times \boldsymbol{J})^{i}(\boldsymbol{r} \times \boldsymbol{J})^{j}+\epsilon^{i j k} m \omega\left(J^{2}-\nu^{2}\right) J_{k} .
$$

In accordance with (2.36), their components satisfy relations

$$
\operatorname{tr}(T)=m\left(J^{2}-\nu^{2}\right) H, \quad \epsilon_{i j k} T^{[j k]}=m \omega\left(J^{2}-\nu^{2}\right) J_{i} .
$$

As the anti-symmetric part of $T^{i j}$ is related with the Poincaré integral, we only need to use the symmetric part $T^{(i j)}$, which is related but not identical to Fradkin's tensor. Since the vectors $(2.30),(2.31)$ are orthogonal to each other and to $\boldsymbol{J}$, we immediately conclude that $\boldsymbol{J}, \boldsymbol{I}_{1}$ and $\boldsymbol{I}_{2}$ are eigenvectors of $T^{(i j)}$ with eigenvalues equal, respectively, to zero and

$$
\begin{aligned}
& \lambda_{1}=\left|\boldsymbol{I}_{1}\right|^{2}=\frac{1}{2} m^{2} \omega^{2}\left(J^{2}-\nu^{2}\right) r_{\max }^{2}, \\
& \lambda_{2}=\left|\boldsymbol{I}_{2}\right|^{2}=\frac{1}{2} m^{2} \omega^{2}\left(J^{2}-\nu^{2}\right) r_{\min }^{2},
\end{aligned}
$$


where we have taken into account (2.35). The relations

$$
\boldsymbol{I}_{1} \cdot \boldsymbol{r}=\left(J^{2}-\nu^{2}\right) \cos (\omega t), \quad \boldsymbol{I}_{2} \cdot \boldsymbol{r}=\left(J^{2}-\nu^{2}\right) \sin (\omega t),
$$

allow us to conclude that the quadratic form $\boldsymbol{r}^{T} T r$ is time-independent,

$$
2 r_{i} T^{i j} r_{j}=\left(\boldsymbol{I}_{1} \cdot \boldsymbol{r}\right)^{2}+\left(\boldsymbol{I}_{2} \cdot \boldsymbol{r}\right)^{2}=\left(J^{2}-\nu^{2}\right)^{2} .
$$

In a coordinate system with orthonormal base $\boldsymbol{e}_{x}=\hat{\boldsymbol{I}}_{1}, \boldsymbol{e}_{y}=\hat{\boldsymbol{I}}_{2}$ and $\boldsymbol{e}_{z}=\hat{\boldsymbol{J}}$, the quadratic form (2.47) simplifies to

$$
\lambda_{1} x^{2}+\lambda_{2} y^{2}=\left(J^{2}-\nu^{2}\right)^{2} .
$$

With $r_{\max } r_{\min }=J /(m \omega)$ one ends up with the equation for an ellipse in the plane orthogonal to $J$ :

$$
\frac{x^{2}}{r_{\min }^{2}}+\frac{y^{2}}{r_{\max }^{2}}=\frac{J^{2}-\nu^{2}}{J^{2}}
$$

The lengths of the semi-major axis and semi-minor axis of the ellipse are $r_{\max } \sqrt{1-\nu^{2} / J^{2}}$, $r_{\min } \sqrt{1-\nu^{2} / J^{2}}$ in accordance with that was found above.

For quantum theory it is of advantage to use the complex form of dynamical integrals of motion

$$
\boldsymbol{a}=\frac{1}{\sqrt{2}}\left(\boldsymbol{I}_{1}+i \boldsymbol{I}_{2}\right)=\boldsymbol{b} \times \boldsymbol{J} e^{i \omega t}, \quad \boldsymbol{b}=\frac{1}{\sqrt{2}}(\boldsymbol{\pi}-i \omega m \boldsymbol{r}),
$$

and its complex conjugate $\boldsymbol{a}^{*}$. They satisfy the non-linear Poisson bracket relations

$$
\begin{aligned}
\{H, \boldsymbol{a}\} & =i \omega \boldsymbol{a}, \quad\left\{J_{i}, a_{j}^{\#}\right\}=\epsilon_{i j k} a_{k}^{\#}, \quad\left\{a_{i}^{\#}, a_{j}^{\#}\right\}=-m \mathcal{C}^{\#} \epsilon_{i j k} J_{k}, \\
\left\{a_{i}^{*}, a_{j}\right\} & \left.=i m \omega\left[\left(2 J^{2}-\nu^{2}\right) \delta_{i j}-J_{i} J_{j}\right)\right]-m H \epsilon_{i j k} J_{k},
\end{aligned}
$$

and are related to the generators (2.22) of the conformal symmetry,

$$
a_{i}^{\#} a_{i}^{\#}=m\left(J^{2}-\nu^{2}\right) \mathcal{C}^{\#},
$$

where $a_{i}^{\#}$ denotes either $a_{i}$ or $a_{i}^{*}$, and similarly for $\mathcal{C}, \mathcal{C}^{*}$. The $a_{i}^{\#}$ and $\mathcal{C}^{\#}$ are classical analogues of the ladder operators in the related quantum system. In terms of $a_{i}^{\#}$ the tensor integrals $T_{(i j)}=T^{(i j)}$ and $T_{[i j]}=T^{[i j]}$ take the form

$$
T_{(i j)}=\frac{1}{2}\left(a_{i}^{*} a_{j}+a_{j}^{*} a_{i}\right), \quad T_{[i j]}=\frac{i}{2}\left(a_{i} a_{j}^{*}-a_{j} a_{i}^{*}\right)=\frac{1}{2} m \omega\left(J^{2}-\nu^{2}\right) \epsilon_{i j k} J_{k} .
$$

In fact, such kind of tensors were considered in earlier studies of the quantized system by Vinet et al. [65]. We find it useful to exploit these integrals for the classical system at hand.

Symmetric tensor integral $T_{(i j)}$ satisfies the Poisson bracket reations

$$
\left\{J_{i}, T_{(j k)}\right\}=\epsilon_{i j l} T_{(l k)}+\epsilon_{i k l} T_{(j l)},
$$

and

$$
\left\{T_{(i j)}, T_{(l k)}\right\}=m\left(\epsilon_{i l s} \mathcal{F}_{j k}+\epsilon_{i k s} \mathcal{F}_{j l}+\epsilon_{j l s} \mathcal{F}_{i k}+\epsilon_{j k s} \mathcal{F}_{i m}\right) J_{s}
$$

where

$$
\mathcal{F}_{i j}=\frac{1}{4} m \omega^{2}\left(J^{2}-\nu^{2}\right)^{2} \delta_{i j}-H T_{(i j)},
$$


and we have used eqs. (2.51), (2.52) and the equality

$$
\mathcal{C}^{*} a_{i} a_{j}+a_{i}^{*} a_{j}^{*} \mathcal{C}=2 H T_{(i j)}+m \omega^{2}\left(J^{2}-\nu^{2}\right)\left[J^{2} \delta_{i j}-J_{i} J_{j}\right] .
$$

In the following section we shall see that the quantum analog of the classical integrals of motion $\mathcal{C}^{\#}$ and $\boldsymbol{a}^{\#}$, due to their dynamical nature of conservation, provide us with a complete set of the spectrum generating operators for the quantum system with Hamiltonian (2.37).

To conclude this section, we comment on the limit $\nu \rightarrow 0$, when we recover the isotropic harmonic oscillator. In this limit the integral $\boldsymbol{a}$ and its complex conjugate reduce to the vector product of the orbital angular momentum and the classical analogs of the first order ladder operators. Instead of considering the dynamical integral $\boldsymbol{a}$ one may choose the vector

$$
\boldsymbol{z}=\left(\boldsymbol{b}+\frac{\nu}{J^{2}}(\boldsymbol{b} \cdot \boldsymbol{n}) \boldsymbol{J}\right) e^{i \omega t}=\frac{1}{J^{2}} \boldsymbol{J} \times \boldsymbol{a}
$$

with $\boldsymbol{b}$ defined in (2.50). This integral and its complex conjugate, which indeed contain the same physical information as $\boldsymbol{a}$ and $\boldsymbol{a}^{\#}$, fulfill the Poisson bracket relations

$$
\left\{z_{i}^{\#}, z_{k}^{\#}\right\}=-\frac{\nu^{2}}{2 J^{4}} \mathcal{C}^{\#} \epsilon_{i k l} J_{l}, \quad\left\{\boldsymbol{z}_{i}, \boldsymbol{z}_{k}^{*}\right\}=-i m \omega \delta_{i, k}+\frac{1}{J^{4}} \mathcal{O}\left(\nu^{2}\right),
$$

where $\mathcal{O}\left(\nu^{2}\right)$ are terms of order $\nu^{2}$. In the limit $\nu \rightarrow 0$ they are just the classical analogs of the first order ladder operators satisfying the Heisenberg algebra. However, the appearance of the non-local operator $1 / J^{2}$ in quantum mechanics complicates the analysis considerably and we prefer to use the integrals (2.50) to deal with local operators in what follows.

\section{Quantum case for $\alpha=\nu^{2}$}

The quantum theory of the system with Hamiltonian (2.37) has been studied earlier in $[65,66]$. Here we reconsider the system as a preparation for our investigation of the related superconformal system with spin-orbit coupling in the next section, and to discuss an interesting relation of the generalized quantum AFF system in the monopole background with its analog without a confining harmonic potential term. First we solve the Schrödinger equation by a separation of variables and afterwards we solve the problem of ladder operators by exploiting the quantum conformal symmetry as well as the hidden symmetry. In a separate subsection, we connect this result with the quantum version of the system $H_{0}$ in (2.28) by construction of a "conformal bridge transformation" following ref. [34]. We shall use the units in which $m=1$ and $\hbar=1$.

In coordinate representation the basic commutation relations are

$$
\left[\hat{r}_{i}, \hat{r}_{j}\right]=0, \quad\left[\hat{r}_{i}, \hat{\pi}_{j}\right]=i \delta_{i j}, \quad\left[\hat{\pi}_{i}, \hat{\pi}_{j}\right]=i \nu \epsilon_{i j k} \frac{\hat{r}_{k}}{r^{3}} .
$$

In what follows we shall skip the hat symbol ^ to simplify the notation. The Hamiltonian (2.37) can be written as

$$
H=\frac{1}{2}\left[-\frac{1}{r^{2}} \frac{\partial}{\partial r}\left(r^{2} \frac{\partial}{\partial r}\right)+\frac{1}{r^{2}} \boldsymbol{J}^{2}+\omega^{2} r^{2}\right]
$$


where $\boldsymbol{J}$ is just the quantum version of the Poincaré integral (2.5), the components of which generate the $\mathfrak{s u}(2)$ symmetry. The Dirac quantization condition implies that $\nu=e g$ must take a integer or half integer value [29-31]. Using the angular momentum treatment we obtain

$$
\boldsymbol{J}^{2} \mathcal{Y}_{j}^{j_{3}}=j(j+1) \mathcal{Y}_{j}^{j_{3}}, \quad J_{3} \mathcal{Y}_{j}^{j_{3}}=j_{3} \mathcal{Y}_{j}^{j_{3}}, \quad J_{ \pm} \mathcal{Y}_{j}^{j_{3}}=c_{j_{3}}^{ \pm} \mathcal{Y}_{j}^{j_{3} \pm 1},
$$

with $J_{ \pm}=J_{1} \pm i J_{2}$, and

$$
j=|\nu|,|\nu|+1, \ldots, \quad j_{3}=-j, \ldots, j, \quad c_{j j_{3}}^{ \pm}=\sqrt{\left(j \pm j_{3}+1\right)\left(j \mp j_{3}\right)},
$$

where the indicated values for $j$ correspond to a super-selection rule. The case $\nu=0$ corresponds just to the quantum harmonic isotropic oscillator. Excluding the zero value for $\nu$, i.e. implying that $|\nu|$ takes any nonzero integer or half-integer value, the first relation in (3.3) automatically provides the necessary inequality $\boldsymbol{J}^{2}=j(j+1)>\nu^{2}$. The functions $\mathcal{Y}_{j}^{j_{3}}=$ $\mathcal{Y}_{j}^{j_{3}}(\theta, \varphi ; \nu)$ are the (normalized) monopole harmonics [29-31, 67-69], which are well defined functions if and only if the combination $j \pm \nu$ is in $\mathbb{N}_{0}=\{0,1,2, \ldots\}$ (see appendix A).

Then, the eigenstates and the spectrum of $H$ are given by

$$
\begin{aligned}
\psi_{n, j}^{j_{3}}(\boldsymbol{r}) & =f_{n, j}(\sqrt{\omega} r) \mathcal{Y}_{j}^{j_{3}}(\theta, \varphi), \\
f_{n, j}(x) & =\left(\frac{2 n !}{\Gamma(n+j+3 / 2)}\right)^{1 / 2} \omega^{3 / 4} x^{j} L_{n}^{(j+1 / 2)}\left(x^{2}\right) e^{-x^{2} / 2}, \\
E_{n, j} & =\left(2 n+j+\frac{3}{2}\right) \omega,
\end{aligned}
$$

where $L_{n}^{(j+1 / 2)}(y)$ are the generalized Laguerre polynomials. The degeneracy of the energy level $E_{n, j}$ can be computed by using the property $E_{n, j}=E_{n+i, j-2 i}$ with $i \in\{-n,-n+$ $1, \ldots,[(j-\nu) / 2]\}$, where [.] is the integer part, and the fact that there are $2(j-2 i)+1$ different states with second index $j-2 i$. This gives us the sought for degeneracy

$$
\mathfrak{g}(\nu, N)=\left\{\begin{array}{l}
\frac{1}{2}(N+\nu+1)(N-\nu+2), j-\nu \text { even } \\
\frac{1}{2}(N-\nu+1)(N+\nu+2), j-\nu \text { odd }
\end{array}, \quad N=2 n+j .\right.
$$

It is remarkable that the system possesses $2|\nu|+1$ degenerate ground states. The ground states here are not invariant under the action of the total angular momentum $\boldsymbol{J}$, although the Hamiltonian operator commutes with $\boldsymbol{J}$ and hence is spherically symmetric. Thus we see some analog of spontaneous breaking of rotational symmetry in the magnetic monopole background. This is of course in contrast to the isotropic harmonic oscillator in three dimensions which has a unique spherically symmetric ground state and symmetry algebra $\mathfrak{s u}(3)$. According to [65] the symmetry algebra for the system under investigation is $\mathfrak{s u}(2) \oplus \mathfrak{s u}(2)$. We do not further dwell on these interesting aspects of symmetry but rather turn to the construction of spectrum generating ladder operators.

Note that the coefficients at radial, $n$, and angular momentum, $j$, quantum numbers in the energy eigenvalue $E_{n, j}=\left(2 n+j+\frac{3}{2}\right) \omega$ correspond to the ratio $P_{a} / P_{r}=l_{a} / l_{r}=2$ 
between the classical angular and radial periods in the special case $\alpha=\nu^{2}$ under investigation. This can be compared with the structure of the principle quantum number $N=n_{r}+l+1$ defining the spectrum in the quantum model of the hydrogen atom, where the corresponding classical periods are equal.

\subsection{The algebraic approach}

The explicit wave functions in (3.5) are specified by the discrete quantum numbers $n, j$ and $j_{3}$. The purpose of this subsection is to identify the ladder operators for radial, $n$, and angular momentum, $j$, quantum numbers (we already have the ladders operators for $j_{3}$ ), which are based on the conformal and hidden symmetries of the system.

In the algebraic approach we do not fix the representation for the position and momentum operators and thus use Dirac's ket notation for eigenstates.

Ladder operators for $\boldsymbol{n}$. Let us first consider the quantum version of the $\mathfrak{s l}(2, \mathbb{R})$ symmetry,

$$
[H, \mathcal{C}]=-2 \omega \mathcal{C}, \quad\left[H, \mathcal{C}^{\dagger}\right]=2 \omega \mathcal{C}^{\dagger}, \quad\left[\mathcal{C}, \mathcal{C}^{\dagger}\right]=4 \omega H,
$$

where the generators $\mathcal{C}, \mathcal{C}^{\dagger}$ are the quantum versions of the integrals (2.22), i.e.

$$
\mathcal{C}=e^{2 i \omega t}\left(H-\omega^{2} r^{2}-\frac{i \omega}{2}(\boldsymbol{r} \cdot \boldsymbol{\pi}+\boldsymbol{\pi} \cdot \boldsymbol{r})\right) .
$$

The time-dependent factors $e^{2 i \omega t}$ in $\mathcal{C}$ and $e^{-2 i \omega t}$ in $\mathcal{C}^{\dagger}$ can be omitted without changing the form of the algebra. Due to the first two equations in (3.7), the scalar nature of $\mathcal{C}$ and $\mathcal{C}^{\dagger}$, and the spectrum (3.5) of the system, it is clear that these operators change $n$ in $n \pm 1$. Then using the relations

$$
\mathcal{C C}^{\dagger}=H^{2}+2 \omega H-\omega^{2}\left(J^{2}-\frac{3}{4}\right), \quad \mathcal{C}^{\dagger} \mathcal{C}=H^{2}-2 \omega H-\omega^{2}\left(J^{2}-\frac{3}{4}\right),
$$

one obtains

$$
\begin{aligned}
\mathcal{C}\left|n, j, j_{3}\right\rangle & =\omega d_{n, j}\left|n-1, j, j_{3}\right\rangle, \quad \mathcal{C}^{\dagger}\left|n, j, j_{3}\right\rangle=\omega d_{n+1, j}\left|n+1, j, j_{3}\right\rangle, \\
d_{n, j} & =\sqrt{2 n(2 n+2 j+1)} .
\end{aligned}
$$

Rescaling the $\mathfrak{s l}(2, R)$ generators, $\mathcal{J}_{0}=\frac{1}{2 \omega} H, \mathcal{J}_{-}=\frac{1}{2 \omega} \mathcal{C}, \mathcal{J}_{+}=\frac{1}{2 \omega} \mathcal{C}^{\dagger}$, the conformal algebra takes the form of the $\mathfrak{s o}(2,1)$ Lorentz algebra

$$
\left[\mathcal{J}_{0}, \mathcal{J}_{ \pm}\right]= \pm \mathcal{J}_{ \pm}, \quad\left[\mathcal{J}_{-}, \mathcal{J}_{+}\right]=2 \mathcal{J}_{0}
$$

with Casimir invariant $\mathscr{F}=-\mathcal{J}_{0}^{2}+\frac{1}{2}\left(\mathcal{J}_{+} \mathcal{J}_{-}+\mathcal{J}_{-} \mathcal{J}_{+}\right)$. From (3.5) it follows that the eigenvalues of $\mathcal{J}_{0}$ in our case are $\mu+n$ with $\mu=\frac{1}{2}\left(j+\frac{3}{2}\right)$, and using (3.9), we find that the Casimir invariant takes on eigenstates (3.5) the value $\mathscr{F}=-\mu(\mu-1)=-\frac{1}{4}\left(j(j+1)-\frac{3}{4}\right)$. If we restore (momentarily) Planck's constant and compare with the classical analog (2.26), we see that the last term in parenthesis is equal to $-\frac{3}{4} \hbar^{2}$ and is a quantum correction. We conclude that each subspace of the Hilbert space of the system characterized by the quantum number $j$ carries an irreducible unitary infinite-dimensional representation of the conformal algebra $\mathfrak{s l}(2, \mathbb{R})$ of the discrete type series $D_{\mu}^{+}[70]$. The operators $\mathcal{C}=2 \omega \mathcal{J}_{-}$ and $C^{\dagger}=2 \omega \mathcal{J}_{+}$correspond here to the ladder operators of $\mathfrak{s l}(2, \mathbb{R})$. 


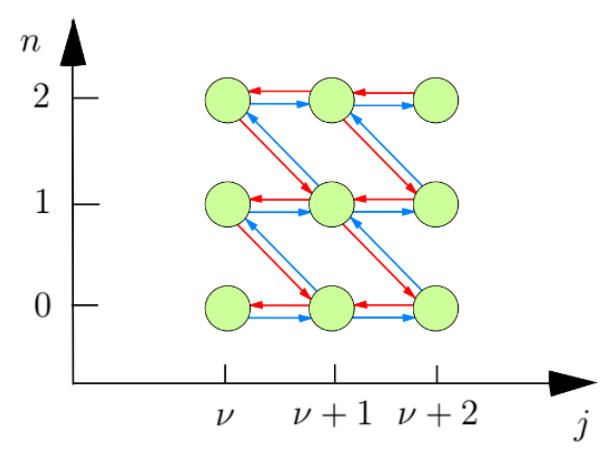

Figure 4. The circles represent the first two quantum numbers of the eigenstates $\left|n, j, j_{3}\right\rangle$. Red arrows indicate the action of $a_{3}$ and blue arrows correspond to the action of $a_{3}^{\dagger}$.

Ladder operators for $\boldsymbol{j}$. We introduce the complex vector operator

$$
\boldsymbol{a}=\frac{1}{2}(\boldsymbol{b} \times \boldsymbol{J}-\boldsymbol{J} \times \boldsymbol{b}) e^{i \omega t}=(\boldsymbol{b} \times \boldsymbol{J}-i \boldsymbol{b}) e^{i \omega t},
$$

together with its Hermitian conjugate, where the vector $\boldsymbol{b}$ has been defined in (2.50). The vector operator $\boldsymbol{a}$ is the quantum version of the complex classical quantity in (2.50) and its components satisfy the relations

$$
\begin{aligned}
{\left[H, a_{i}\right] } & =-\omega a_{i}, \quad\left[J_{i}, a_{j}\right]=i \epsilon_{i j k} a_{k}, \quad\left[a_{i}, a_{j}\right]=-i \epsilon_{i j k} \mathcal{C} J_{k}, \\
{\left[a_{i}^{\dagger}, a_{j}\right] } & \left.=-\omega\left[\left(2 J^{2}+1-\nu^{2}\right) \delta_{i j}-J_{i} J_{j}\right)\right]-i H \epsilon_{i j k} J_{k},
\end{aligned}
$$

with corresponding relations for the Hermitian conjugate $a_{i}^{\dagger}$. Again, the time-dependent phase $e^{i \omega t}$ can be omitted, i.e., one can make a change $\boldsymbol{a} \rightarrow \boldsymbol{a} e^{-i \omega t}$ accompanied by the analogous omission of the time-dependent phase in the generators of the conformal algebra, that does not change the form of the commutation relations (3.14) and (3.15).

The action of these operators is computed algebraically in appendix B. Here it is sufficient to consider $a_{3}$ and $a_{3}^{\dagger}$ and their actions on the ket-states

$$
\begin{aligned}
& a_{3}\left|n, j, j_{3}\right\rangle=A_{n, j, j_{3}}\left|n, j-1, j_{3}\right\rangle+B_{n, j, j_{3}}\left|n-1, j+1, j_{3}\right\rangle, \\
& a_{3}^{\dagger}\left|n, j, j_{3}\right\rangle=A_{n, j+1, j_{3}}\left|n, j+1, j_{3}\right\rangle+B_{n+1, j-1, j_{3}}\left|n+1, j-1, j_{3}\right\rangle,
\end{aligned}
$$

where the squares of the positive coefficients are

$$
\left(A_{n, j, j_{3}}\right)^{2}=\omega(2 n+2 j+1) \frac{\left(j^{2}-j_{3}^{2}\right)\left(j^{2}-\nu^{2}\right)}{(2 j)^{2}-1}, \quad\left(B_{n, j, j_{3}}\right)^{2}=\frac{2 n}{2 n+2 j+3}\left(A_{n, j+1, j_{3}}\right)^{2} .
$$

We see that the operators $a_{3}$ and $a_{3}^{\dagger}$ change the quantum numbers $n$ and $j$, but the result is a superposition of the two eigenstate vectors. Their action is depicted in figure 4. Clearly, it would be preferable to find ladder operators that map a given eigenstate into just one eigenstate with a different quantum number $j$ and not a superposition of eigenstates. To find such operators we introduce the non-local operator

$$
\mathscr{J}=\sqrt{\boldsymbol{J}^{2}+\frac{1}{4}}-\frac{1}{2}, \quad \mathscr{J}\left|n, j, j_{3}\right\rangle=j\left|n, j, j_{3}\right\rangle
$$


and construct the operators

$$
\mathscr{T}_{ \pm}=\omega\left(\mathscr{J}+\frac{1}{2}\right) a_{3} \pm(H-\omega) a_{3} \mp a_{3}^{\dagger} \mathcal{C}
$$

together with their Hermitean conjugate. Actually $\mathscr{T}_{ \pm}$and $\mathscr{T}_{ \pm}^{\dagger}$ are the third components of the vector operators $\mathcal{T}_{ \pm}$and $\mathcal{T}_{ \pm}^{\dagger}$ which are given by (3.20) wherein $a_{3}$ and $a_{3}^{\dagger}$ are replaced by $\boldsymbol{a}$ and $\boldsymbol{a}^{\dagger}$ on the right hand side. But in what follows it suffices to consider $\mathscr{T}_{ \pm}$and $\mathscr{T}_{ \pm}^{\dagger}$ which are ladder operators for the energy,

$$
\left[H, \mathscr{T}_{ \pm}\right]=\omega \mathscr{T}_{ \pm}, \quad\left[H, \mathscr{T}_{ \pm}^{\dagger}\right]=-\omega \mathscr{T}_{ \pm}^{\dagger}
$$

They decrease and increase the angular momentum according to

$$
\begin{aligned}
\mathscr{T}_{+}\left|n, j, j_{3}\right\rangle & =\omega(2 j+1) A_{n, j, j_{3}}\left|n, j-1, j_{3}\right\rangle, \\
\mathscr{T}_{-}\left|n, j, j_{3}\right\rangle & =\omega(2 j+3) B_{n, j, j_{3}}\left|n-1, j+1, j_{3}\right\rangle,
\end{aligned}
$$

and the analogous Hermitian conjugate relations. These non-local objects were inspired by a similar construction presented in [59] for the three dimensional isotropic harmonic oscillator.

Now one can generate in a simple way all eigenstates of the commuting observables $H, \boldsymbol{J}^{2}$ and $J_{3}$ by acting with the local ladder operators $\mathcal{C}, \mathcal{C}^{\dagger}, J_{ \pm}$and with the non-local ladder operators $\mathscr{T}_{+}, \mathscr{T}_{+}^{\dagger}$ on just one eigenstate. The same can be achieved with local ladder operators when one uses $a, a^{\dagger}$ instead of $\mathscr{T}_{+}, \mathscr{T}_{+}^{\dagger}$, but then the recursive construction get more involved, since $a, a^{\dagger}$ map into a superposition of eigenstates.

\subsection{The conformal bridge}

Here we show how the generators of the conformal as well as the hidden symmetry of the quantum system (3.2) can be obtained from generators of the corresponding symmetries of the quantum system studied in [32]. This will be realized by means of the special nonunitary transformation considered recently in [34] and identified there as a "conformal bridge transformation". As it will be seen, such a transformation simultaneously generates eigenstates and coherent states of the system (3.2) from certain states of the quantum system considered in [32].

Similarly to the classical case, in the limit $\omega \rightarrow 0$ the quantum version of the generators (2.24) has the form

$$
\begin{aligned}
& H_{0}=\frac{1}{2}\left(\boldsymbol{\pi}^{2}+\frac{\nu^{2}}{r^{2}}\right)=\frac{1}{2}\left(-\frac{1}{r^{2}} \frac{\partial}{\partial r}\left(r^{2} \frac{\partial}{\partial r}\right)+\frac{1}{r^{2}} \boldsymbol{J}^{2}\right), \\
& D_{0}=\frac{1}{4}(\boldsymbol{r} \boldsymbol{\pi}+\boldsymbol{\pi} \cdot \boldsymbol{r})-H_{0} t, \quad K_{0}=\frac{1}{2} r^{2}-D t-H_{0} t^{2} .
\end{aligned}
$$

They produce the quantum conformal algebra

$$
\left[D_{0}, H_{0}\right]=i H_{0}, \quad\left[D_{0}, K_{0}\right]=-i K_{0}, \quad\left[K_{0}, H_{0}\right]=2 i D_{0} .
$$


The Hamiltonian $H_{0}$ is a non-compact generator of the conformal algebra $\mathfrak{s l}(2, \mathbb{R})$ with a continuos spectrum $(0, \infty)$. In the same limit, the quantum version of the vector integrals $\boldsymbol{I}_{1}$ and $\boldsymbol{I}_{2}$ transforms into the vectors

$$
\left.\boldsymbol{I}_{1} \rightarrow \frac{1}{2}(\boldsymbol{\pi} \times \boldsymbol{J}-\boldsymbol{J} \times \boldsymbol{\pi}):=\boldsymbol{V}, \quad \frac{\boldsymbol{I}_{2}}{\omega} \rightarrow \frac{1}{2}(\boldsymbol{\pi} t-\boldsymbol{r}) \times \boldsymbol{J}-\boldsymbol{J} \times(\boldsymbol{\pi} t-\boldsymbol{r})\right):=\boldsymbol{G},
$$

which we identify, respectively, as the Laplace-Runge-Lentz vector and the Galilei boost generator for the system $H_{0}$ [32] in the Weyl-ordered form. The commutator relations of the vectors $\boldsymbol{V}$ and $\boldsymbol{G}$ with the generators of the conformal algebra are

$$
\begin{array}{rlrl}
{\left[H_{0}, G_{i}\right]} & =-i V_{i}, & {\left[K_{0}, V_{i}\right]=i G_{i},} & {\left[H_{0}, V_{i}\right]=\left[K_{0}, G_{i}\right]=0} \\
{\left[D_{0}, V_{i}\right]=\frac{i}{2} V_{i},} & {\left[D_{0}, G_{i}\right]=-\frac{i}{2} G_{i} .} &
\end{array}
$$

In order to go in the opposite direction, i.e., to recover our system $H$ and its symmetry generators starting from the generators (3.24), (3.25) and (3.27), we implement a particular non-unitary transformation. The conformal bridge transformation [34] corresponds to setting $t=0$ in the definition of the generators above and in the construction of the operator

$$
\mathfrak{S}=e^{-\omega K_{0}} e^{\frac{1}{2 \omega} H_{0}} e^{i \ln 2 D_{0}}
$$

in terms of the conformal symmetry generators of the system $H_{0}$. A similarity transformation generated by $\mathfrak{S}$ yields

$$
\begin{aligned}
\mathfrak{S} \boldsymbol{J} \mathfrak{S}^{-1} & =\boldsymbol{J}, & \mathfrak{S} \boldsymbol{V} \mathfrak{S}^{-1} & =\boldsymbol{a}, & \mathfrak{S} \omega \boldsymbol{G} \mathfrak{S}^{-1} & =-i \boldsymbol{a}^{\dagger} \\
\mathfrak{S}\left(H_{0}\right) \mathfrak{S}^{-1} & =\frac{1}{2} \mathcal{C}, & \mathfrak{S}\left(2 i \omega D_{0}\right) \mathfrak{S}^{-1} & =H, & \mathfrak{S}\left(\omega^{2} K_{0}\right) \mathfrak{S}^{-1} & =-\frac{1}{2} \mathcal{C}^{\dagger}
\end{aligned}
$$

where $H=H_{0}+\omega^{2} K_{0}$ is the quantum Hamiltonian (3.2). The explicit time dependence can be recovered then by applying a unitary transformation with the evolution operator $U=e^{-i H t}$.

In correspondence with the second relation in (3.32), the wave functions (3.5) can be generated, up to a normalization, by applying the non-unitary operator $\mathfrak{S}$ to the formal eigenstates of the first order differential operator $2 i \omega D_{0}$ defined by the equation $2 i \omega D_{0} \chi_{n, j}^{j_{3}}=\omega(2 n+j+3 / 2) \chi_{n, j}^{j_{3}}$. The latter are given by

$$
\chi_{n, j}^{j_{3}}(r, \theta, \phi)=r^{j+2 n} \mathcal{Y}_{j}^{j_{3}}(\theta, \phi) .
$$

To see that $\mathfrak{S}$ has these properties, we consider the following relation which can be proved by induction,

$$
\left(H_{0}\right)^{\ell} \chi_{n, j}^{j_{3}}(\boldsymbol{r})=\frac{(-2)^{\ell} \Gamma(n+1) \Gamma(n+j+3 / 2)}{\Gamma(n+1-\ell) \Gamma(n+j+3 / 2-\ell)} \chi_{n-\ell, j}^{j_{3}}, \quad \ell=1,2, \ldots
$$

It vanishes for $\ell>n$ due to the pole of the Gamma function in the denominator. Therefore, $\chi_{0, j}^{j_{3}}$ can be interpreted as the zero-energy eigenstate of $H_{0}$, and $\chi_{n, j}^{j_{3}}, n=1,2, \ldots$, are its 
Jordan states corresponding to the same zero energy [71]. Decomposing the operator $\exp \left(\frac{1}{2 \omega} H_{0}\right)$ entering the definition of $\mathfrak{S}$ and using equation (3.34) one obtains

$$
\mathfrak{S} \chi_{n, j}^{j_{3}}=\frac{(-1)^{n}}{\sqrt{2}}\left(\frac{2}{\omega}\right)^{n+\frac{j}{2}+\frac{3}{4}}[n ! \Gamma(n+j+3 / 2)]^{\frac{1}{2}} \psi_{n, j}^{j_{3}} .
$$

On the other hand, one can show that solutions of the equation $H_{0} \phi_{j}^{j_{3}}=\frac{1}{2} \kappa^{2} \phi_{j}^{j_{3}}$ are mapped into coherent states of $H$. The explicit form of these solutions is given by

$$
\phi_{j}^{j 3}(\boldsymbol{r} ; \kappa)=\frac{1}{\sqrt{r}} J_{j+\frac{1}{2}}(\kappa r) \mathcal{Y}_{j}^{j_{3}}=\sum_{n=0}^{\infty} \frac{(-1)^{n}(\kappa / 2)^{2 n+j+1 / 2}}{n ! \Gamma(n+j+3 / 2)} \chi_{n, j}^{j_{3}}(\boldsymbol{r}),
$$

where in the first equality $J_{j+\frac{1}{2}}(y)$ is the Bessel function of the first kind, and in the second one we show that the solution can be expressed as a series expansion in terms of the Jordan states (3.34). The explicit action of $\mathfrak{S}$ on (3.36) gives us

$$
\begin{aligned}
\zeta_{j}^{j_{3}}(\boldsymbol{r} ; \kappa) & =N \mathfrak{S} \phi_{j}^{j 3}\left(\boldsymbol{r} ; \frac{\kappa}{\sqrt{2}}\right)=\sqrt{2} N e^{-\frac{\omega x^{2}}{2}+\frac{\kappa^{2}}{4 \omega}} \phi_{j}^{j 3}(\boldsymbol{r} ; \kappa) \\
& =\frac{N}{\omega^{1 / 2}} \sum_{n=0}^{\infty} \frac{1}{\sqrt{n ! \Gamma\left(n+j+\frac{3}{2}\right)}}\left(\frac{\kappa}{2 \sqrt{\omega}}\right)^{2 n+j+1 / 2} \psi_{n, j}^{j_{3}}(\boldsymbol{r}),
\end{aligned}
$$

where $N$ stand for a normalization constant. Using the series expansion in terms of $\psi_{n, j}^{j_{3}}$, one finds $N^{2}=\sqrt{\omega} /\left(I_{j+\frac{1}{2}}\left(\frac{|\kappa|^{2}}{2 \omega}\right)\right)$, where $I_{j+\frac{1}{2}}(z)$ is the modified Bessel function of the first kind, and we have put the modulus in its argument because $\kappa$ admits an analytic extension for complex values, as is usual for coherent states. Also, this expansion helps us to find the time evolution of these functions generated by $H$ as follows,

$$
\zeta_{j}^{j_{3}}(\boldsymbol{r}, t ; \kappa)=e^{-i H t} \zeta_{j}^{j_{3}}(\boldsymbol{r} ; \kappa)=e^{-i \frac{\omega}{2} t} \zeta_{j}^{j_{3}}\left(\boldsymbol{r} ; \kappa e^{-\frac{i \omega t}{2}}\right) .
$$

At the same time, the first equation in (3.32) shows us that $\zeta_{j}^{j_{3}}(\boldsymbol{r}, t ; \kappa)$ are eigenstates of $\mathcal{C}$ with eigenvalue $-\frac{1}{2} \kappa^{2} e^{-i \omega t}$, i.e. they are indeed the coherent states of the system corresponding to the conformal algebra (3.7) [72].

\section{A charge-monopole superconformal model}

In this section we admit an additional contribution to the Hamiltonian (3.2) due to the spin degrees of freedom of the particle. The additional term describes a strong long-range spin-orbit coupling and gives rise to an exactly solvable supersymmetric extension of the three-dimensional system studied in previous sections. After introducing the system in the first subsection we analyze its peculiar properties. In particular, we will find that some energy values are infinitely degenerate and others are not. In the second subsection, we show how one can extend the model to a supersymmetric system by means of the factorization method, and by using this we construct an explicit realization of the $\mathfrak{o s p}(2 \mid 2)$ superconformal symmetry. In the third subsection we show that two different superconformal extensions of the one-dimensional AFF model with unbroken and spontaneously broken phases of $\mathcal{N}=2$ Poincaré supersymmetry can be obtained by reduction from our three-dimensional $\mathfrak{o s p}(2 \mid 2)$ superconformal system. 


\subsection{Spin-orbit coupling model}

Let us consider the following two Hamiltonians with strong spin-orbit coupling

$$
H_{ \pm \omega}=\frac{1}{2}\left(\boldsymbol{\pi}^{2}+\omega^{2} r^{2}+\frac{\nu^{2}}{r^{2}}\right) \pm \omega \boldsymbol{\sigma} \cdot \boldsymbol{J}=H \pm \omega \boldsymbol{\sigma} \cdot \boldsymbol{J} .
$$

The Hamiltonians $H_{ \pm \omega}$ are similar to those which appear as subsystems of the nonrelativistic limit of the supersymmetric Dirac oscillator discussed in $[57,58]$. Thus the eigenvalue problems can be solved similarly as in those references, but the usual spherical harmonics are replaced by the monopole harmonics. Actually, if we would choose a spinorbit coupling $\omega^{\prime} \boldsymbol{\sigma} \cdot \boldsymbol{J}$ with $0 \leq \omega<\omega^{\prime}$, then the spectra of both Hamiltonians would be unbounded from below. On the other hand, for $0 \leq \omega^{\prime}<\omega$ all energies will have finite degeneracy. Only in the very particular case $\omega^{\prime}=\omega$, which we consider here, the spectra are bounded from below and half of the energies have a finite degeneracy whereas the other half have infinite degeneracy. This reminds us the BPS-limits in field theory, where different interactions balance and supersymmetry is observed.

The operators $H$ and $\boldsymbol{\sigma} \cdot \boldsymbol{J}$ commute and as a consequence $H_{ \pm \omega}$ commute with the "total angular momentum"

$$
\boldsymbol{K}=\boldsymbol{J}+\boldsymbol{s}=\boldsymbol{J}+\frac{1}{2} \boldsymbol{\sigma}, \quad\left[K_{i}, K_{j}\right]=i \epsilon_{i j k} K_{k} .
$$

The possible eigenvalues of $\boldsymbol{K}^{2}$ are $k(k+1)$. It is well-known how to construct the simultaneous eigenstates of $\boldsymbol{K}^{2}$ and $K_{3}$ :

$$
\left|n, k, k_{3}, \pm\right\rangle=\sum_{m_{s}} C_{j j_{3} \frac{1}{2} m_{s}}^{k k_{3}}\left|n, j, j_{3}\right\rangle \otimes\left|\frac{1}{2}, m_{s}\right\rangle_{k=j \pm \frac{1}{2}},
$$

where the Clebsch-Gordan coefficients

$$
C_{j j_{3} \frac{1}{2} m_{s}}^{k k_{3}}=\left\langle j, j_{3}, \frac{1}{2}, m_{s} \mid k, k_{3}\right\rangle
$$

on the right hand side are nonzero only if $j_{3}+m_{s}=k_{3}$ and if the triangle-rule holds, which means that the total angular momentum $k$ is either $j+\frac{1}{2}$ or $j-\frac{1}{2}$. In the first case the eigenstates of the total angular momentum are denoted by $\left|\ldots, k, k_{3},+\right\rangle$ and in the second case by $\left|\ldots, k, k_{3},-\right\rangle$. The sums (4.3) contain just two terms, since the eigenvalue $m_{s}$ of the third spin-component $s_{3}=\frac{1}{2} \sigma_{3}$ is either $\frac{1}{2}$ or $-\frac{1}{2}$. Note that in the coordinate representation the wavefunctions corresponding to these kets are given in (3.5), i.e.

$$
\begin{aligned}
\left\langle\boldsymbol{r} \mid n, k, k_{3}, \pm\right\rangle & =f_{n, j}(\sqrt{\omega} r)\left\langle\boldsymbol{n} \mid k, k_{3}, \pm\right\rangle \\
\left\langle\boldsymbol{n} \mid k, k_{3}, \pm\right\rangle & =\frac{1}{\sqrt{2 k+1 \mp 1}}\left(\begin{array}{c} 
\pm \sqrt{k \pm k_{3}+(1 \mp 1) / 2} \mathcal{Y}_{k \mp 1 / 2}^{k_{3}-1 / 2}(\theta, \varphi ; \nu) \\
\sqrt{k \mp k_{3}+(1 \mp 1) / 2} \mathcal{Y}_{k \mp 1 / 2}^{k_{3}+1 / 2}(\theta, \varphi ; \nu)
\end{array}\right):=\Omega_{k}^{k_{3} \pm} .
\end{aligned}
$$

The wavefunctions (4.5) contain the monopole harmonics and generalized Laguerre polynomials. If $\nu=e g$ is integer-valued then $j$ is a non-negative integer and $k$ a positive half-integer. If $e g$ is half-integer, then $j$ is a positive half-integer and $k$ is in $\mathbb{N}_{0}$. 
The vector in (4.3) is a simultaneous eigenstate of $\boldsymbol{J}^{2}$ with eigenvalue $j(j+1)$, of $\boldsymbol{K}^{2}$ with eigenvalue $k(k+1)$, of $H$ with eigenvalue $\left(2 n+j+\frac{3}{2}\right) \omega$, where $j=k \mp 1 / 2$, and finally of the operator $\boldsymbol{\sigma} \cdot \boldsymbol{J}$ :

$$
\boldsymbol{\sigma} \cdot \boldsymbol{J}\left|n, k, k_{3}, \pm\right\rangle=\left( \pm\left(k+\frac{1}{2}\right)-1\right)\left|n, k, k_{3}, \pm\right\rangle
$$

As a consequence the action of the Hamiltonians in (4.1) on these states is

$$
\begin{aligned}
& H_{+\omega}\left|n, k, k_{3}, \pm\right\rangle=\omega\left(2 n+k+\frac{1}{2} \pm k\right)\left|n, k, k_{3}, \pm\right\rangle, \\
& H_{-\omega}\left|n, k, k_{3}, \pm\right\rangle=\omega\left(2 n+k+\frac{5}{2} \mp(k+1)\right)\left|n, k, k_{3}, \pm\right\rangle .
\end{aligned}
$$

We see that the discrete eigenvalues of both Hamiltonians $H_{ \pm \omega}$ fall into two families: in one family all energies are infinitely degenerate and in the other family they all have finite degeneracy (due to their dependence on the quantum number $k$ ). More explicitly, for $k=j \mp \frac{1}{2}$ the eigenvalues of $H_{\mp \omega}$ have infinite degeneracy and for $k=j \pm \frac{1}{2}$ they have finite degeneracy $\mathfrak{g}(N, \nu)=N^{2}-\nu^{2}$, where $N=n+j+1$. A similar peculiar behavior is observed in the Dirac oscillator spectrum [57].

Operators $K_{ \pm}=K_{1} \pm i K_{2}$ are the ladder operators for the magnetic quantum number $k_{3}$. The ladder operators for the radial quantum number are given in (3.8), and their action on the simultaneous eigenstates reads

$$
\begin{aligned}
\mathcal{C}\left|n, k, k_{3}, \pm\right\rangle & =\omega d_{n, j}\left|n-1, k, k_{3}, \pm\right\rangle \\
\mathcal{C}^{\dagger}\left|n, k, k_{3}, \pm\right\rangle & =\omega d_{n+1, j}\left|n+1, k, k_{3}, \pm\right\rangle
\end{aligned}
$$

with coefficients defined in (3.11). Thus, as for the spin-zero particle system in monopole background, we can easily construct local ladder operators for $n$ and $k_{3}$. But again, finding ladder operators for $k$ is more difficult. One way to proceed is to follow the ideas employed for the Dirac oscillator in [59, 60]. First we decompose the total Hilbert space in two subspaces, $\mathscr{H}=\mathscr{H}^{(+)} \oplus \mathscr{H}^{(-)}$, where each $\mathscr{H}^{( \pm)}$is spanned by the states $\left|n, k, k_{3}, \pm\right\rangle$. Actually we can construct non-local operators which project orthonormally onto these subspaces,

$$
\begin{aligned}
& \mathscr{P}_{+}=\frac{1}{2}+\sqrt{\boldsymbol{K}^{2}+\frac{1}{4}}-\sqrt{\boldsymbol{J}^{2}+\frac{1}{4}}, \\
& \mathscr{P}_{-}=\frac{1}{2}-\sqrt{\boldsymbol{K}^{2}+\frac{1}{4}}+\sqrt{\boldsymbol{J}^{2}+\frac{1}{4}},
\end{aligned}
$$

and reproduce or annihilate the eigenstates,

$$
\left.\mathscr{P}_{( \pm)}\right|_{\mathscr{H}^{( \pm)}}=\left.\mathbb{1}\right|_{\mathscr{H}^{( \pm)}},\left.\quad \mathscr{P}_{( \pm)}\right|_{\mathscr{H}^{(\mp)}}=0
$$

In next step we introduce the operators

$$
\mathcal{A}_{ \pm}=\mathscr{P}_{ \pm} \mathscr{T}_{ \pm} \mathscr{P}_{ \pm}
$$


where the non-local $\mathscr{T}_{ \pm}$have been defined in (3.20). The presence of the projectors will ensure that $\mathcal{A}_{ \pm}$only acts on eigenstates in $\mathscr{H}^{( \pm)}$, and its action on these eigenstates can be computed straightforwardly using the relations (3.22) and (3.23):

$$
\begin{aligned}
\mathcal{A}_{+}\left|n, k, k_{3},+\right\rangle & =(k-1) \sqrt{n+k} \Lambda_{k, k_{3}, j}\left|n, k-1, k_{3},+\right\rangle, \\
\mathcal{A}_{-}\left|n, k-1, k_{3},-\right\rangle & =(k+1) \sqrt{n} \Lambda_{k, k_{3}, j}\left|n-1, k, k_{3},-\right\rangle,
\end{aligned}
$$

with

$$
\Lambda_{k, k_{3}, j}=\frac{\omega^{3 / 2}}{k} \sqrt{2\left(k^{2}-k_{3}^{2}\right)\left(j^{2}-\nu^{2}\right)} .
$$

These relations mean that the operators $\mathcal{A}_{ \pm}$and their adjoint act as ladder operators for the quantum number $k$. Together with operators $K_{ \pm}, \mathcal{C}, \mathcal{C}^{\dagger}$ they generate all eigenstates in the full Hilbert space from just two eigenstates, one from each subspace $\mathscr{H}^{( \pm)}$.

\subsection{The $\mathfrak{o s p}(2 \mid 2)$ superconformal extension}

In this subsection we construct and analyze supersymmetric partners of the Hamiltonians $H_{ \pm \omega}$ by introducing factorizing operators. From these we obtain two $\mathcal{N}=2$ super-Poincaré quantum systems which are related to each other by a common integral of motion which generates an $R$-symmetry. Supplementing the supercharges of one of these systems by supercharges of another, we extend the $\mathcal{N}=2$ super-Poincaré symmetry up to the $\mathfrak{o s p}(2 \mid 2)$ superconformal symmetry realized by a three-dimensional system of spin- $1 / 2$ particle in a monopole background.

Consider the first-order scalar operators

$$
\Theta=i \boldsymbol{\sigma} \cdot \boldsymbol{b}-\frac{1}{\sqrt{2}} \frac{\nu}{r}, \quad \Xi=i \boldsymbol{\sigma} \cdot \boldsymbol{b}^{\dagger}-\frac{1}{\sqrt{2}} \frac{\nu}{r},
$$

and their adjoint $\Theta^{\dagger}$ and $\Xi^{\dagger}$. The products of these operators with their adjoint are

$$
H_{[1]}:=\Theta \Theta^{\dagger}=H_{+\omega}+\frac{3}{2} \omega, \quad \breve{H}_{[1]}:=\Xi \Xi^{\dagger}=H_{-\omega}-\frac{3}{2} \omega,
$$

where $H_{ \pm \omega}$ are given in (4.1). The associated superpartners take the form

$$
\begin{aligned}
& H_{[0]}:=\Theta^{\dagger} \Theta=\breve{H}_{[1]}-\nu\left(\frac{1}{r^{2}}+2 \omega\right) \sigma_{r}, \\
& \breve{H}_{[0]}:=\Xi^{\dagger} \Xi=H_{[1]}-\nu\left(\frac{1}{r^{2}}-2 \omega\right) \sigma_{r},
\end{aligned}
$$

wherein the projection of $\boldsymbol{\sigma}$ to the normal unit vector appears,

$$
\sigma_{r}=\boldsymbol{n} \cdot \boldsymbol{\sigma}=\left(\begin{array}{cc}
\cos \theta & e^{-i \varphi} \sin \theta \\
e^{i \varphi} \sin \theta & -\cos \theta
\end{array}\right)
$$

The first order operators satisfy the intertwining relations

$$
\begin{array}{ll}
\Theta H_{[0]}=H_{[1]} \Theta, & \Theta^{\dagger} H_{[1]}=H_{[0]} \Theta^{\dagger}, \\
\Xi \breve{H}_{[0]}=\breve{H}_{[0]} \Xi, & \Xi^{\dagger} \breve{H}_{[1]}=\breve{H}_{[0]} \Xi^{\dagger} .
\end{array}
$$


The eigenstates of $H_{[0]}$ can be obtained by acting with $\Theta^{\dagger}$ on the eigenstates of $H_{[1]}$ or equivalently of $H_{+\omega}$. They are given in (4.3). For computing the action of the intertwining operators we use the following representation

$$
\Theta^{\dagger}=\frac{\sigma_{r}}{\sqrt{2}}\left(-\frac{1}{r} \frac{\partial}{\partial r} r+\omega r+\frac{1+\boldsymbol{\sigma} \cdot \boldsymbol{J}}{r}\right)
$$

As a result we obtain the relations

$$
\left.\Theta^{\dagger}\left|n, k, k_{3}, \pm\right\rangle= \pm \sqrt{2 \omega\left(n+1+\beta_{ \pm} k\right)} \| n+\beta_{\mp}, k, k_{3}, \pm\right\rangle, \quad \beta_{ \pm}=\frac{1}{2}(1 \pm 1),
$$

where in coordinate representation the normalized spinors $\left.\| n, k, k_{3}, \pm\right\rangle$ on the right hand side have the explicit form

$$
\left\langle\boldsymbol{r} \| n, k, k_{3}, \pm\right\rangle=f_{n, j \pm 1} \sigma_{r} \Omega_{k}^{k_{3}} \pm,
$$

where $\Omega_{k}^{k_{3} \pm}$ are given in (4.6). With the help of functional relations between different generalized Laguerre polynomials (see appendix $\mathrm{C}$ ) one proves that

$$
\left.\Theta \| n, k, k_{3}, \pm\right\rangle= \pm \sqrt{2 \omega\left(n+\beta_{ \pm}(k+1)\right)}\left|n-\beta_{\mp}, k, k_{3}, \pm\right\rangle,
$$

and by acting with the operator $\Theta^{\dagger}$ on these relations we get the eigenvalue equations

$$
\left.\left.H_{[0]} \| n, k, k_{3}, \pm\right\rangle=2 \omega\left(n+\beta_{ \pm}(k+1)\right) \| n, k, k_{3}, \pm\right\rangle .
$$

Note that the states $\left.\| n, k, k_{3},-\right\rangle$ are zero-modes of $H_{[0]}$ since they are annihilated by $\Theta$.

The eigenstates of $\breve{H}_{[0]}$ can be determined in an analogous way by acting with the operator

$$
\Xi^{\dagger}=\frac{\sigma_{r}}{\sqrt{2}}\left(-\frac{1}{r} \frac{\partial}{\partial r} r-\omega r+\frac{1}{r}(1+\boldsymbol{\sigma} \cdot \boldsymbol{J})\right)
$$

on the spinors (4.5) and with its adjoint $\Xi$ on the spinors (4.27), that results in the mappings

$$
\begin{aligned}
\Xi^{\dagger}\left|n, k, k_{3}, \pm\right\rangle & \left.= \pm \sqrt{2 \omega\left(n+\beta_{\mp}(k+1)\right.} \| n-\beta_{ \pm}, k, k_{3}, \pm\right\rangle, \\
\left.\Xi \| n, k, k_{3}, \pm\right\rangle & = \pm \sqrt{2 \omega\left(n+1+\beta_{\mp} k\right)}\left|n+\beta_{ \pm}, k, k_{3}, \pm\right\rangle .
\end{aligned}
$$

Note that $\Xi^{\dagger}$ as well as $\breve{H}_{[1]}$ annihilate the set of states $\left.\| 0, k, k_{3},+\right\rangle$.

Finally, acting with $\Xi^{\dagger}$ on the states in (4.32), we solve the eigenvalues problem for $\breve{H}_{[0]}$ :

$$
\left.\left.\breve{H}_{[0]} \| n, k, k_{3}, \pm\right\rangle=2 \omega\left(n+1+k \beta_{\mp}\right) \| n, k, k_{3}, \pm\right\rangle .
$$

Having at hand the eigenstates $\left.\| n, k, k_{3}, \pm\right\rangle$, one may find spectrum generating ladder operators. In this context equations (4.26), (4.28), (4.31) and (4.32) can be used to construct such operators for the quantum number $n$. They read

$$
\tilde{\mathcal{C}}=\Xi^{\dagger} \Theta, \quad \tilde{\mathcal{C}}^{\dagger}=\Theta^{\dagger} \Xi
$$

and act on the eigenvectors $\| \ldots\rangle$ as follows:

$$
\begin{aligned}
\left.\tilde{\mathcal{C}}^{\dagger} \| n, k, k_{3}, \pm\right\rangle & \left.=2 \omega d_{n+1, j \pm 1} \| n+1, k, k_{3}, \pm\right\rangle \\
\left.\tilde{\mathcal{C}} \| n, k, k_{3}, \pm\right\rangle & \left.=2 \omega d_{n, j \pm 1} \| n-1, k, k_{3}, \pm\right\rangle
\end{aligned}
$$


Actually, the first order operators $\Theta$ and $\Xi^{\dagger}$ factorize the earlier considered second order ladder operator (3.8) according to $\mathcal{C}=\Theta \Xi^{\dagger}$.

Having constructed lowering and raising operators for $n$, we are still missing ladder operators for $k$ and $k_{3}$. For the latter we may of course use $K_{ \pm}$, since $\Theta, \Xi$ and their adjoint are scalar operators with respect to $\boldsymbol{K}$. But once more, for the angular momentum quantum number $k$ we can introduce non-local "dressed" operators

$$
\tilde{\mathcal{A}}_{-}=\Theta \sqrt{\frac{1}{H_{[1]}}} \mathcal{A}_{-} \sqrt{\frac{1}{H_{[1]}}} \Theta^{\dagger}, \quad \tilde{\mathcal{A}}_{+}=\Xi \sqrt{\frac{1}{\breve{H}_{[1]}}} \mathcal{A}_{+} \sqrt{\frac{1}{\breve{H}_{[1]}}} \Xi^{\dagger},
$$

and their adjoint operators, where $\mathcal{A}_{ \pm}$have been given in (4.15). The operators $\tilde{\mathcal{A}}_{ \pm}$are the analogs to $\mathcal{A}_{ \pm}$for the vectors $\left.\| n, k, k_{3}, \pm\right\rangle$, as we see in equations

$$
\begin{aligned}
\left.\tilde{\mathcal{A}}_{+} \| n, k, k_{3},+\right\rangle & \left.=(k-1) \sqrt{n+k} \Lambda_{k, k_{3}, j} \| n, k-1, k_{3},+\right\rangle, \\
\left.\tilde{\mathcal{A}}_{-} \| n, k-1, k_{3},-\right\rangle & \left.=(k+1) \sqrt{n} \Lambda_{k, k_{3}, j} \| n-1, k, k_{3},-\right\rangle .
\end{aligned}
$$

In a final step we combine the four $2 \times 2$ matrix Hamiltonians introduced above into two $4 \times 4$ matrix super-Hamiltonians as follows:

$$
\mathcal{H}=\left(\begin{array}{cc}
H_{[1]} & 0 \\
0 & H_{[0]}
\end{array}\right), \quad \breve{\mathcal{H}}=\left(\begin{array}{cc}
\breve{H}_{[1]} & 0 \\
0 & \breve{H}_{[0]}
\end{array}\right) .
$$

In the limit $\nu \rightarrow 0$ they turn into different versions of the Dirac oscillator in the nonrelativistic limit, see [57]. Both operators commute with the $\mathbb{Z}_{2}$-grading operator $\Gamma=$ $\sigma_{3} \otimes \mathbb{I}_{2 \times 2},[\Gamma, \mathcal{H}]=[\Gamma, \breve{\mathcal{H}}]=0$, and their difference is the (bosonic) integral of motion

$$
\mathcal{R}=\frac{1}{2 \omega}(\mathcal{H}-\breve{\mathcal{H}})=\left(\boldsymbol{J} \cdot \boldsymbol{\sigma}+\frac{3}{2}\right) \Gamma-2 \nu \sigma_{r} \Pi_{-}=\left(\begin{array}{cc}
\boldsymbol{\sigma} \cdot \boldsymbol{J}+\frac{3}{2} & 0 \\
0 & -\left(\boldsymbol{\sigma} \cdot \boldsymbol{J}+2 \nu \sigma_{r}+\frac{3}{2}\right)
\end{array}\right)
$$

where $\Pi_{-}$is a projector,

$$
\Pi_{ \pm}=\frac{1}{2}(1 \pm \Gamma)
$$

In the fermionic sectors of the systems $\mathcal{H}$ and $\breve{\mathcal{H}}$ we have the nilpotent operators

$$
\mathcal{Q}=\left(\begin{array}{ll}
0 & \Theta \\
0 & 0
\end{array}\right), \quad \mathcal{W}^{\dagger}=\left(\begin{array}{ll}
0 & \Xi \\
0 & 0
\end{array}\right)
$$

$\{\Gamma, \mathcal{Q}\}=\{\Gamma, \mathcal{W}\}=0$, and their adjoint operators. Each of these generate an $\mathcal{N}=2$ Poincaré superalgebra

$$
\begin{array}{rlrl}
{[\mathcal{H}, \mathcal{Q}]} & =\left[\mathcal{H}, \mathcal{Q}^{\dagger}\right]=\{\mathcal{Q}, \mathcal{Q}\}=\left\{\mathcal{Q}^{\dagger}, \mathcal{Q}^{\dagger}\right\}=0, & \left\{\mathcal{Q}, \mathcal{Q}^{\dagger}\right\} & =\mathcal{H}, \\
{[\breve{\mathcal{H}}, \mathcal{W}]} & =\left[\breve{\mathcal{H}}, \mathcal{W}^{\dagger}\right]=\{\mathcal{W}, \mathcal{W}\}=\left\{\mathcal{W}^{\dagger}, \mathcal{W}^{\dagger}\right\}=0, & \left\{\mathcal{W}, \mathcal{W}^{\dagger}\right\}=\breve{\mathcal{H}}
\end{array}
$$

The even integral $\mathcal{R}$ in (4.40) generates an $R$-symmetry for both systems, and satisfies the relations (for details see appendix D),

$$
[\Gamma, \mathcal{R}]=0, \quad[\mathcal{R}, \mathcal{Q}]=\mathcal{Q}, \quad[\mathcal{R}, \mathcal{W}]=-\mathcal{W}, \quad \text { h.c. }
$$


where h.c. corresponds to Hermitian conjugate relations. Having in mind that $\mathcal{H}$ and $\breve{\mathcal{H}}$ can be diagonalized simultaneously, from now on we treat $\mathcal{H}$ as the Hamiltonian of the super-extended system and $\breve{\mathcal{H}}=\mathcal{H}-2 \omega \mathcal{R}$ as its integral. Then, by anti-commuting $\mathcal{Q}$ and $\mathcal{W}$ we obtain the bosonic generator

$$
\mathcal{G}=\{\mathcal{W}, \mathcal{Q}\}=\left(\begin{array}{cc}
\mathcal{C} & 0 \\
0 & \tilde{\mathcal{C}}
\end{array}\right), \quad[\Gamma, \mathcal{G}]=0, \quad \text { h.c. }
$$

composed from the ladder operators of sub-systems $H_{[1]}$ and $H_{[0]}$ of our system $\mathcal{H}$.

Taking together, these scalar generators with respect to

$$
\mathcal{K}_{i}=\left(\begin{array}{cc}
K_{i} & 0 \\
0 & K_{i}
\end{array}\right), \quad i=1,2,3,
$$

obey the superalgebraic relations

$$
\begin{aligned}
{[\mathcal{H}, \mathcal{G}] } & =-2 \omega \mathcal{G}, & {\left[\mathcal{G}, \mathcal{G}^{\dagger}\right] } & =4 \omega(\mathcal{H}-\omega \mathcal{R}), \\
{[\mathcal{H}, \mathcal{W}] } & =-2 \omega \mathcal{W}, & {[\mathcal{R}, \mathcal{W}] } & =-\mathcal{W}, \\
{\left[\mathcal{G}, \mathcal{Q}^{\dagger}\right] } & =-2 \omega \mathcal{W}, & {\left[\mathcal{G}, \mathcal{W}^{\dagger}\right] } & =2 \omega \mathcal{Q}, \\
\left\{\mathcal{Q}, \mathcal{Q}^{\dagger}\right\} & =\mathcal{H}, & \left\{\mathcal{W}, \mathcal{W}^{\dagger}\right\} & =(\mathcal{H}-2 \omega \mathcal{R}), \\
\{\mathcal{Q}, \mathcal{W}\} & =\mathcal{G}, & &
\end{aligned}
$$

supplemented by the adjoint relations. The (anti)-commutators not displayed here do vanish. This superalgebra is identified as the $\mathfrak{o s p}(2 \mid 2)$ superconformal symmetry which appears in systems like one-dimensional harmonic super-oscillator or the superconformal mechanics model with a confining term $[19,20,71,73]$. Therefore our construction maybe considered as generalization of the three-dimensional versions of these systems in the monopole background.

The relations (4.48)-(4.52) are invariant under the automorphism $\mathcal{H} \rightarrow \breve{\mathcal{H}}, \mathcal{R} \rightarrow \mathcal{R}$, $\mathcal{Q} \leftrightarrow \mathcal{W}, \mathcal{G} \rightarrow \mathcal{G}$ and h.c., which amount to using $\breve{\mathcal{H}}$ instead of $\mathcal{H}$, as super-Hamiltonian. The common eigenstates of $\mathcal{H}, \mathcal{R}, \Gamma, \mathcal{K}_{3}$ and $\mathcal{K}^{2}$ are given by

$$
\left|n, k, k_{3}, \pm, 1\right\rangle=\left(\begin{array}{c}
\left|n, k, k_{3}, \pm\right\rangle \\
0
\end{array}\right), \quad\left|n, k, k_{3}, \pm,-1\right\rangle=\left(\begin{array}{c}
0 \\
\left.\| n, k, k_{3}, \pm\right\rangle
\end{array}\right)
$$

which satisfy the eigenvalue equations

$$
\begin{aligned}
\mathcal{H}\left|n, k, k_{3}, \pm, \gamma\right\rangle & =2 \omega\left(n+\frac{1}{2}(1+\gamma)+\beta_{ \pm}\left(k+\frac{1}{2}(1-\gamma)\right)\right)\left|n, k, k_{3}, \pm, \gamma\right\rangle \\
\Gamma\left|n, k, k_{3}, \pm, \gamma\right\rangle & =\gamma\left|n, k, k_{3}, \pm, \gamma\right\rangle, \quad \gamma= \pm 1 \\
\mathcal{R}\left|n, k, k_{3}, \pm, \gamma\right\rangle & =\left[ \pm\left(k+\frac{1}{2}\right)+\frac{\gamma}{2}\right]\left|n, k, k_{3}, \pm, \gamma\right\rangle \\
\mathcal{K}^{2}\left|n, k, k_{3}, \pm, \gamma\right\rangle & =k(k+1)\left|n, k, k_{3}, \pm, \gamma\right\rangle \\
\mathcal{K}_{3}\left|n, k, k_{3}, \pm, \gamma\right\rangle & =k_{3}\left|n, k, k_{3}, \pm, \gamma\right\rangle .
\end{aligned}
$$


The operators $\mathcal{Q}$ and $\mathcal{Q}^{\dagger}\left(\mathcal{W}\right.$ and $\left.\mathcal{W}^{\dagger}\right)$ defined in (4.42), interchange the state vectors $\left|n, k, k_{3}, \pm, \gamma\right\rangle$ and $\left|n, k, k_{3}, \pm,-\gamma\right\rangle$ according to the rules in (4.26), (4.28) and (4.31), (4.32). The ground states of $\mathcal{H}(\breve{\mathcal{H}})$ which are given by $\left|n, k, k_{3},-,-1\right\rangle\left(\left|n, k, k_{3},+,+1\right\rangle\right)$ are invariant under transformations generated by these fermionic operators, therefore the quantum system $\mathcal{H}$ exhibits the unbroken $\mathcal{N}=2$ Poincaré supersymmetry.

Finally, the spectrum generating ladder operators for the supersymmetric system correspond to operators $\mathcal{G}$ and $\mathcal{G}^{\dagger}$ for $n, \mathcal{K}_{ \pm}$for $k_{3}$ and the matrix non-local operators

$$
\left(\begin{array}{cc}
\mathcal{A}_{ \pm} & 0 \\
0 & \tilde{\mathcal{A}}_{ \pm}
\end{array}\right), \quad\left(\begin{array}{cc}
\mathcal{A}_{ \pm}^{\dagger} & 0 \\
0 & \tilde{\mathcal{A}}_{ \pm}^{\dagger}
\end{array}\right) .
$$

for the angular quantum number $k$.

\subsection{Dimensional reduction}

In this section we trace out how two different super-extensions of the one-dimensional AFF model can be obtained by a reduction from our three-dimensional superconformal system. For the sake of simplicity we put here $\omega=1$, and denote $\sqrt{\omega} r=r$ as $x$.

Let us revisit first the supersymmetric AFF model. There are two possible extensions, which are given by the $2 \times 2$ matrix Hamiltonians

$$
\mathcal{H}_{\ell}^{\epsilon}=\left(\begin{array}{cc}
H_{\ell}+\epsilon\left(\ell-\frac{1}{2}\right) & 0 \\
0 & H_{\ell-1}+\epsilon\left(\ell+\frac{1}{2}\right)
\end{array}\right), \quad H_{\ell}=\frac{1}{2}\left(-\frac{d^{2}}{d x^{2}}+x^{2}+\frac{\ell(\ell+1)}{x^{2}}\right),
$$

where $\epsilon= \pm$ and $\ell \geq-1 / 2$ [74-77]. The $\mathbb{Z}_{2}$-grading operator is $\sigma_{3}$, and the supercharges of super-extended systems $\mathcal{H}_{\ell}^{\epsilon}$ are given by

$$
Q_{\ell, 1}^{\epsilon}=-\frac{\epsilon}{\sqrt{2}}\left(\begin{array}{cc}
0 & A_{\ell}^{\epsilon} \\
A_{\ell}^{\epsilon \dagger} & 0
\end{array}\right), \quad Q_{\ell, 2}^{\epsilon}=i \sigma_{3} Q_{\ell, 1}^{\epsilon},
$$

where

$$
A_{\ell}^{\epsilon}=-\epsilon \frac{d}{d x}+x+\epsilon \frac{\ell}{x} .
$$

The supercharges and Hamilltonian operators satisfy the $\mathcal{N}=2$ Poincaré superalgebra

$$
\left\{Q_{\ell, a}^{\epsilon}, Q_{\ell, b}^{\epsilon}\right\}=2 \delta_{a b} \mathcal{H}_{\ell}^{\epsilon}, \quad\left[\mathcal{H}_{\ell}^{\epsilon}, Q_{\ell, a}^{\epsilon}\right]=0, \quad a, b=1,2 .
$$

As in the case studied in the previous section, here we can also construct the $R$ symmetry generator

$$
R_{\ell}=\frac{1}{2}\left(\mathcal{H}_{\ell}^{-}-\mathcal{H}_{\ell}^{+}\right)=\frac{1}{2} \sigma_{3}-\ell,
$$

and therefore one Hamiltonian can be expressed in terms of another and $R_{\ell}$. Additionally, we have the conformal symmetry ladder operator

$$
\mathfrak{G}_{\ell}=\left(\begin{array}{cc}
G_{\ell} & 0 \\
0 & G_{\ell-1}
\end{array}\right), \quad G_{\ell}=-\frac{1}{2}\left(\frac{d}{d x}+x\right)^{2}+\frac{\ell(\ell+1)}{2 x^{2}},
$$


and its adjoint, which are generated by

$$
\left\{Q_{\ell, a}^{\epsilon}, Q_{\ell, b}^{-\epsilon}\right\}=\delta_{a b}\left(\mathfrak{G}_{\ell}+\mathfrak{G}_{\ell}^{\dagger}\right)+i \epsilon_{a b}\left(\mathfrak{G}_{\ell}-\mathfrak{G}_{\ell}^{\dagger}\right) .
$$

By constructing nilpotent fermionic operators $\frac{1}{2}\left(Q_{\ell, a}^{\epsilon} \pm i Q_{\ell, a}^{\epsilon}\right)$, it is not difficult to show that these generators satisfy the algebra (4.48)-(4.52).

The eigenstates of the super-Hamiltonian $\mathcal{H}_{\ell}^{\epsilon}$ and supercharge $Q_{\ell, 1}^{\epsilon}$, which we will denote as $\Phi_{n, \ell, \varrho}^{\epsilon}$ with $\varrho= \pm 1$, are given by

$$
\begin{aligned}
& \Phi_{0, \ell, 1}^{-}=\frac{x}{\sqrt{2}}\left(\begin{array}{c}
0 \\
f_{0, \ell-1}(x)
\end{array}\right), \\
& \Phi_{n+1, \ell, 1}^{-}=\frac{x}{\sqrt{2}}\left(\begin{array}{c}
f_{n, \ell}(x) \\
f_{n+1, \ell-1}(x)
\end{array}\right), \quad \Phi_{n+1, \ell,-1}^{-}=\sigma_{3} \Phi_{n+1, \ell, 1}^{-}, \\
& \Phi_{n, \ell, 1}^{+}=\frac{x}{\sqrt{2}}\left(\begin{array}{c}
f_{n, \ell}(x) \\
f_{n, \ell-1}(x)
\end{array}\right), \quad \Phi_{n, \ell,-1}^{+}=\sigma_{3} \Phi_{n, \ell, 1}^{+}, \quad n \in \mathbb{N}_{0} .
\end{aligned}
$$

The spectral equations are

$$
\mathcal{H}_{\ell}^{\epsilon} \Phi_{n, \ell, \varrho}^{\epsilon}=\left(2 n+\beta_{ \pm}(2 \ell+1)\right) \Phi_{n, \ell, \varrho}^{\epsilon}, \quad Q_{\ell, 1}^{\epsilon} \Phi_{n, \ell, \varrho}^{-}=\varrho \sqrt{\left(2 n+\beta_{\epsilon}(2 \ell+1)\right)} \Phi_{n, \ell, \varrho}^{\epsilon},
$$

where $\beta_{+}=1, \beta_{-}=0$, cf. (4.26). In the case of $\mathcal{H}_{\ell}^{-}$, the ground state $\Phi_{0, \ell, 1}^{-}$is annihilated by the super-Hamiltonian and by the supercharges $Q_{\ell, a}^{-}$, and, therefore, supersymmetry is unbroken, with energy levels being independent of $\ell$. On the other hand, $\mathcal{H}_{\ell}^{+}$has no zero-energy ground state, energy levels depend on parameter $\ell$, and there is no physical eigenstate annihilated by both supercharges $Q_{\ell, a}^{+}$, that implies that supersymmetry is spontaneously broken. For more details see refs. [19, 20, 71]. The independence and dependence of energy levels on $\ell$ is reminiscent of two subsets of states in our three-dimensional system with infinitely degenerate energy levels due to their independence on the quantum number $j$ and finitely degenerate, depending on $j$ energy eigenvalues. This is an additional indication on that one-dimensional superconformal extensions of the AFF model (4.60) may indeed be obtained by reduction from our three-dimensional $\mathfrak{o s p}(2 \mid 2)$ superconformal system.

In the following, we will show that for two different dimensional reductions of the system $\mathcal{H}$ defined in (4.39), we obtain a particular realization of the one-dimensional superextended AFF model in both, broken and unbroken, $\mathcal{N}=2$ supersymmetry phases, with $\ell=j$ taking one of the values $j=|\nu|,|\nu|+1 \ldots$ To this end we first note that the Hamiltonian $\mathcal{H}$ admits a representation

$$
\mathcal{H}=\frac{1}{2}\left[-\frac{1}{x^{2}} \frac{\partial}{\partial x}\left(x^{2} \frac{\partial}{\partial x}\right)+x^{2}\right] \mathbb{I}_{4 \times 4}+\frac{1}{2 x^{2}}\left(\mathcal{K}^{2}-\Gamma \mathcal{R}+\frac{3}{4}\right)+\mathcal{R} .
$$

Also, let us introduce the following notation to distinguish one-dimensional from threedimensional generators:

$$
\begin{aligned}
\mathscr{B}_{j, \alpha}^{\epsilon} & =\left\{\mathcal{H}_{j}^{\epsilon}, R_{j}, \mathfrak{G}_{j}, \mathfrak{G}_{j}^{\dagger}\right\}, & \mathscr{F}_{j, \beta}^{\epsilon} & =\left\{Q_{j, 1}^{\epsilon}, Q_{j, 2}^{\epsilon}, Q_{j, 1}^{-\epsilon}, Q_{j, 2}^{-\epsilon}\right\}, \\
\mathcal{B}_{\alpha} & =\left\{\mathcal{H}, \mathcal{R}_{j}, \mathcal{G}_{j}, \mathcal{G}_{j}^{\dagger}\right\}, & \mathcal{F}_{\beta} & =\{\operatorname{Re}(\mathcal{Q}), \operatorname{Im}(\mathcal{Q}), \operatorname{Re}(\mathcal{W}), \operatorname{Im}(\mathcal{W})\},
\end{aligned}
$$

where we imply that $\mathcal{B}_{j, 1}=\mathcal{H}$ etc., and $\operatorname{Re}(\mathcal{Q})=\frac{1}{2}\left(\mathcal{Q}+\mathcal{Q}^{\dagger}\right), \operatorname{Im}(\mathcal{Q})=\frac{i}{2}\left(\mathcal{Q}^{\dagger}-\mathcal{Q}\right)$. 
For the dimensional reduction we "extract" a subspace in which the angular and spin operators in (4.71) take fixed numerical values. We have two independent choices which we distinguish by the signs \pm , and they relate to the choice of the states $|\chi, \pm\rangle$ defined by the set of equations

$$
\begin{aligned}
\left(\mathcal{K}^{2}-k(k+1)\right)|\chi, \pm\rangle & =0, & & \left(\mathcal{K}_{3}-k_{3}\right)|\chi, \pm\rangle=0 \\
\mathcal{P}_{ \pm}|\chi, \pm\rangle & =0, & & \mathcal{P}_{ \pm}=\frac{1}{2 k+1}\left(\Pi_{ \pm}+k \mp \mathcal{R}\right)
\end{aligned}
$$

where $k=j \pm \frac{1}{2}$, and $k_{3}=j_{3} \pm \frac{1}{2}$. Here, the most general form of $|\chi, \pm\rangle$ is

$$
|\chi, \pm\rangle=\sum_{n=0}^{\infty} a_{n}^{ \pm}\left|n, k, k_{3}, \pm, 1\right\rangle+b_{n}^{ \pm}\left|n, k, k_{3}, \pm,-1\right\rangle=\sum_{n=0}^{\infty}\left(\begin{array}{l}
a_{n}^{ \pm}\left|n, k, k_{3}, \pm\right\rangle \\
\left.b_{n}^{ \pm} \| n, k, k_{3}, \pm\right\rangle
\end{array}\right) .
$$

The operators $\mathcal{P}_{ \pm}$are projectors onto the orthogonal subspaces $|\chi,-\rangle$ and $|\chi,+\rangle$. In both subspaces, the grading operator preserves its form, while the action of operators $\mathcal{R}$ and $\mathcal{K}^{2}-\Gamma \mathcal{R}+3 / 4$ produce

$$
\begin{aligned}
\mathcal{R}|\chi,-\rangle & =\left(\begin{array}{cc}
-\left(j-\frac{1}{2}\right) \mathbb{I}_{2 \times 2} & 0 \\
0 & -\left(j+\frac{1}{2}\right) \mathbb{I}_{2 \times 2}
\end{array}\right)|\chi,-\rangle \\
& =R_{j} \otimes \mathbb{I}_{2 \times 2}|\chi,-\rangle, \\
\mathcal{R}|\chi,+\rangle & =\left(\begin{array}{cc}
\left(j+\frac{3}{2}\right) \mathbb{I}_{2 \times 2} & 0 \\
0 & \left(j+\frac{1}{2}\right) \mathbb{I}_{2 \times 2}
\end{array}\right)|\chi,+\rangle \\
& =-\sigma_{1}\left(R_{j+1}\right) \sigma_{1} \otimes \mathbb{I}_{2 \times 2}|\chi,+\rangle, \\
\left(\mathcal{K}^{2}-\Gamma \mathcal{R}+\frac{3}{4}\right)|\chi, \pm\rangle & =\left(\begin{array}{cc}
j(j+1) & 0 \\
0 & j(j \pm 1)
\end{array}\right) \otimes \mathbb{I}_{2 \times 2}|\chi, \pm\rangle,
\end{aligned}
$$

where the generator $R_{j}$ defined in (4.64) appears explicitly. In the same way we found in the subspace represented by $|\chi,-\rangle$ the following relations,

$$
\mathcal{B}_{a}|\chi,-\rangle=\frac{1}{x} \mathscr{B}_{j}^{-} x \otimes \mathbb{I}_{2 \times 2}|\chi,-\rangle, \quad \mathcal{F}_{b}|\chi,-\rangle=\frac{1}{x} \mathscr{F}_{j}^{-} x \otimes \sigma_{r}|\chi,-\rangle,
$$

while in the subspace given by $|\chi,+\rangle$ we obtain

$$
\mathcal{B}_{a}|\chi,+\rangle=\sigma_{1}\left(\frac{1}{x} \mathscr{B}_{j+1}^{+} x\right) \sigma_{1} \otimes \mathbb{I}_{2 \times 2}|\chi,+\rangle, \quad \mathcal{F}_{b}|\chi,+\rangle=\sigma_{1}\left(\frac{1}{x} \mathscr{F}_{j}^{+} x\right) \sigma_{1} \otimes \sigma_{r}|\chi,+\rangle .
$$

In these equations the generators take the form of a direct product of two operators $A \otimes B$, where $A$ is a one-dimensional $2 \times 2$ matrix operator, and $B$ is the $2 \times 2$ identity matrix or $\sigma_{r}$. The latter still contains an angular dependence, see (4.22). To eliminate the angular variables we introduce the operators

$$
\mathcal{O}_{ \pm}=\left(\begin{array}{cc}
|v\rangle\left\langle k, k_{3}, \pm\right| & 0 \\
0 & |v\rangle\left\langle k, k_{3}, \pm\right| \sigma_{r}
\end{array}\right), \quad|v\rangle=\left(\begin{array}{l}
1 \\
1
\end{array}\right)
$$


and their adjoints. Here $\left|k, k_{3}, \pm\right\rangle$ corresponds to (4.5). Acting on the state $|\chi, \pm\rangle$, these operators produce

$$
\mathcal{O}_{ \pm}|\chi, \pm\rangle=|\Psi, \pm\rangle, \quad\langle r \mid \Psi, \pm\rangle=\sum_{n=0}^{\infty}\left(\begin{array}{c}
a_{n}^{ \pm} f_{n, j} \\
a_{n}^{ \pm} f_{n, j} \\
b_{n}^{ \pm} f_{n, j \pm 1} \\
b_{n}^{ \pm} f_{n, j \pm 1}
\end{array}\right)
$$

and $\left(\mathcal{O}_{ \pm}\right)^{\dagger}|\Psi, \pm\rangle=|\chi, \pm\rangle$, that implies that $\left(\mathcal{O}_{ \pm}\right)^{\dagger} \mathcal{O}_{ \pm}|\chi, \pm\rangle=|\chi, \pm\rangle$ and $\mathcal{O}_{ \pm}\left(\mathcal{O}_{ \pm}\right)^{\dagger}|\Psi, \pm\rangle=|\Psi, \pm\rangle$. Multiplication of the bosonic generators by $\mathcal{O}_{ \pm}$from the left and by $\mathcal{O}_{ \pm}^{\dagger}$ from the right does not change their structure, i.e. $\mathcal{O}_{ \pm} \mathcal{B}_{\alpha}\left(\mathcal{O}_{ \pm}\right)^{\dagger}=\mathcal{B}_{\alpha}$, but the same operation applied to fermionic generators produces

$$
\begin{aligned}
\mathcal{O}_{-} \mathcal{F}_{b} \mathcal{O}_{-}^{\dagger}|\Psi,-\rangle & =\frac{1}{x} \mathscr{F}_{j, \beta}^{-} x \otimes \sigma_{1}|\Psi,-\rangle, \\
\mathcal{O}_{+} \mathcal{F}_{b} \mathcal{O}_{+}^{\dagger}|\Psi,+\rangle & =\sigma_{1}\left(\frac{1}{x} \mathscr{F}_{j+1, \beta}^{+} x\right) \sigma_{1} \otimes \sigma_{1}|\Psi,+\rangle .
\end{aligned}
$$

Note that $\sigma_{r}$ disappears, and we effectively eliminated the angular degrees of freedom. The reduction scheme is almost done. To complete it we introduce the unitary matrix

$$
U=\left(\begin{array}{llll}
1 & 0 & 0 & 0 \\
0 & 0 & 1 & 0 \\
0 & 1 & 0 & 0 \\
0 & 0 & 0 & 1
\end{array}\right), \quad U U^{\dagger}=1, \quad \operatorname{det} U=-1,
$$

which finally gives

$$
\begin{aligned}
U \mathcal{B}_{a}|\Psi,-\rangle & =\frac{1}{x}\left(\begin{array}{cc}
\mathscr{B}_{j, a}^{-} & 0 \\
0 & \mathscr{B}_{j, a}^{-}
\end{array}\right) x U|\Psi,-\rangle, \\
U \mathcal{O}_{-} \hat{\mathcal{F}}_{\beta} \mathcal{O}_{-}^{\dagger}|\Psi,-\rangle & =\frac{1}{x}\left(\begin{array}{cc}
0 & \mathscr{F}_{j, \beta}^{-} \\
\mathscr{F}_{j, \beta}^{-} & 0
\end{array}\right) x U|\Psi,-\rangle, \\
U \mathcal{B}_{a}|\Psi,+\rangle & =\frac{1}{x}\left(\begin{array}{cc}
\sigma_{1} \mathscr{B}_{j+1, a}^{+} \sigma_{1} & 0 \\
0 & \sigma_{1} \mathscr{B}_{j+1, a}^{+} \sigma_{1}
\end{array}\right) x U|\Psi,+\rangle, \\
U \mathcal{O}_{+} \hat{\mathcal{F}}_{\beta} \mathcal{O}_{+}^{\dagger}|\Psi,+\rangle & =\frac{1}{x}\left(\begin{array}{cc}
0 & \sigma_{1} \mathscr{F}_{j+1, \beta}^{+} \sigma_{1} \\
\sigma_{1} \mathscr{F}_{j+1, \beta}^{+} \sigma_{1} & 0
\end{array}\right) x U|\Psi,+\rangle,
\end{aligned}
$$

where each of these matrices is a $4 \times 4$ matrix, and

$$
\langle r|U| \Psi, \pm\rangle=\sum_{n=0}^{\infty}\left(\begin{array}{c}
a_{n}^{ \pm} f_{n, j} \\
b_{n}^{ \pm} f_{n, j \pm 1} \\
a_{n}^{ \pm} f_{n, j} \\
b_{n}^{ \pm} f_{n, j \pm 1}
\end{array}\right)
$$


The last state contains two copies of the same two-component column vector, which in turn can be expanded in terms of eigenstates (4.67), (4.68) divided by $x$ in the case when we do the reduction with sign -, or in terms of the states (4.69) multiplied by $\sigma_{1} / x$, if we choose the sign + . On the other hand, in equations (4.87)-(4.90) particular bosonic (fermionic) generators appear as block-(anti)diagonal matrices, where each block corresponds to the same one-dimensional generator. To eliminate one of these copies we can use the projector operators $\Pi_{ \pm}$. Then we obtain

$$
\begin{aligned}
\left\langle\boldsymbol{r}\left|\Pi_{ \pm} U \mathcal{B}_{\alpha}\right| \Psi,-\right\rangle & \rightarrow \mathscr{B}_{j, \alpha}^{-} \Psi_{j}^{-}(x), \quad\left\langle\boldsymbol{r}\left|\Pi_{ \pm} U \mathcal{B}_{\alpha}\right| \Psi,+\right\rangle \rightarrow \sigma_{1} \mathscr{B}_{j+1, \alpha}^{+} \Psi_{j+1}^{+}(x), \\
\left\langle\boldsymbol{r}\left|\Pi_{ \pm} U \mathcal{O}_{-} \mathcal{F}_{\alpha}\left(\mathcal{O}_{-}\right)^{\dagger}\right| \Psi,-\right\rangle & \rightarrow \mathscr{F}_{j, \beta}^{-} \Psi_{j}^{-}(x) \\
\left\langle\boldsymbol{r}\left|\Pi_{ \pm} U \mathcal{O}_{+} \mathcal{F}_{\alpha}\left(\mathcal{O}_{+}\right)^{\dagger}\right| \Psi,+\right\rangle & \rightarrow \sigma_{1} \mathscr{F}_{j+1, \beta}^{+} \Psi_{j+1}^{+}(x)
\end{aligned}
$$

where

$$
\begin{gathered}
\Psi_{j}^{-}=x\left\langle\boldsymbol{r}\left|\Pi_{ \pm} U \mathcal{O}_{-}\right| \chi,-\right\rangle=\sum_{n=0}^{\infty} A_{n}^{-} \Phi_{n, j, 1}^{-}+B_{n}^{-} \Phi_{n, j,-1}^{-}, \\
\Psi_{j+1}^{+}=x \sigma_{1}\left\langle\boldsymbol{r}\left|\Pi_{ \pm} U \mathcal{O}_{+}\right| \chi,+\right\rangle=\sum_{n=0}^{\infty} A_{n}^{+} \Phi_{n, j+1,1}^{+}+B_{n}^{+} \Phi_{n, j+1,-1}^{+} .
\end{gathered}
$$

and the coefficients $A_{n}^{ \pm}$and $B_{n}^{ \pm}$can be expressed in terms of $a_{n}^{ \pm}$and $b_{n}^{ \pm}$in (4.76) using the orthogonality of states (4.67)-(4.69) with $\ell=j$.

Thus, the appropriately realized dimensional reduction of our three-dimensional $\mathfrak{o s p}(2 \mid 2)$ superconformal system $\mathcal{H}$ with the unbroken $\mathcal{N}=2$ Poincaré supersymmetry produces two different $\mathfrak{o s p}(2 \mid 2)$ superconformal extensions of the one-dimensional AFF model with unbroken or spontaneously broken $\mathcal{N}=2$ Poincaré supersymmetries.

\section{Discussion and outlook}

In summary, this work is divided in two parts. In the first part, we studied the special case of a dynamical conformal system presented by a scalar charged particle in the monopole background which is characterized by the presence of an additional, hidden symmetry that controls and reflects its peculiar classical and quantum properties. In the second part, we added spin degrees of freedom by introducing a spin-orbit coupling of a special, unique form that guarantees a very peculiar degeneracy of energy levels and gives rise to the superconformal $\mathfrak{o s p}(2 \mid 2)$ symmetry. By two different dimensional reduction schemes this three-dimensional supersymmetric system produces the one-dimensional superconformal extensions of the AFF model [44] in unbroken and spontaneously broken phases of $\mathcal{N}=2$ Poincaré supersymmetry [20].

The scalar charged particle in the monopole background that we considered is subjected to a central potential $V(r)=\frac{\alpha}{2 m r^{2}}+\frac{m \omega^{2}}{2} r^{2}$, which is a three-dimensional analog of the AFF model's potential, and therefore the system posseses the conformal Newton-Hooke symmetry [61-64]. For coupling constant $\alpha \neq \nu^{2}$, trajectories are closed only for some particular initial conditions. On the contrary, the special case $\alpha=\nu^{2}$ we study always gives 
us closed trajectories, the angular period is twice the radial period, and even more, the dynamics projected to the plane orthogonal to the Poincaré angular momentum vector $\boldsymbol{J}$ turns out to be similar to that for the usual three-dimensional isotropic harmonic oscillator. In fact, such an interesting "coincidence" is a universal property of the monopole background. ${ }^{3}$ Indeed, if we consider the system described by the Hamiltonian

$$
H_{\nu}=\frac{\pi^{2}}{2 m}+\frac{\nu^{2}}{2 m r^{2}}+U(r)
$$

with an arbitrary central potential $U(r)$, then the dynamics of the vector variables $\boldsymbol{r} \times \boldsymbol{J}$ and $\boldsymbol{\pi} \times \boldsymbol{J}$ has the same form as that for vector variables $\boldsymbol{r} \times \boldsymbol{L}$ and $\boldsymbol{p} \times \boldsymbol{L}$ when $\nu=e g=0$ with $\boldsymbol{L}$ being the usual angular momentum:

$$
\begin{array}{|c|c|}
\hline \nu \neq 0 & \nu=0 \\
\hline \frac{d}{d t}(\boldsymbol{r} \times \boldsymbol{J})=\frac{1}{m} \boldsymbol{\pi} \times \boldsymbol{J} & \frac{d}{d t}(\boldsymbol{r} \times \boldsymbol{L})=\frac{1}{m} \boldsymbol{p} \times \boldsymbol{L} \\
\hline \frac{d}{d t}(\boldsymbol{\pi} \times \boldsymbol{J})=U^{\prime}(r) \boldsymbol{n} \times \boldsymbol{J} & \frac{d}{d t}(\boldsymbol{p} \times \boldsymbol{L})=U^{\prime}(r) \boldsymbol{n} \times \boldsymbol{L} \\
\hline
\end{array}
$$

As a consequence, the motion in the plane orthogonal to $J$ is equivalent to the dynamics obtained in the absence of the monopole, and if we know the solutions $r=r(t)$ and $\boldsymbol{p}=\boldsymbol{p}(t)$ in the case $\nu=0$, the dynamics for $\boldsymbol{\pi} \times \boldsymbol{J}$ and $\boldsymbol{r} \times \boldsymbol{J}$ is at hand. To reconstruct the complete dynamics we combine the relations (2.39) to obtain

$$
\boldsymbol{r}(t)=\frac{1}{J^{2}}\left(\boldsymbol{J} \times(\boldsymbol{r}(t) \times \boldsymbol{J})+\sqrt{\frac{|\boldsymbol{r}(t) \times \boldsymbol{J}|}{J^{2}-\nu^{2}} \boldsymbol{J}}\right)
$$

In particular, if instead of (5.1) we have a system described by the Hamiltonian $\widetilde{H}_{\nu}=$ $\frac{1}{2 m} \boldsymbol{\pi}^{2}+\widetilde{U}(r)$ with arbitrary central potential $\widetilde{U}(r)$, it is reduced in an obvious way to the system (5.1) with central potential $U(r)=\widetilde{U}(r)-\nu^{2} / 2 m r^{2}$. The indicated similarity and relation allows, particularly, to identify immediately the analog of the Laplace-Runge-Lenz vector (3.27) for a particle in the monopole background in the case of $\widetilde{U}=0$ and $U=0$, and for the Kepler problem with $U=q / r$, that was done earlier in [30-32] and [65] but by using a different approach.

From this perspective, one can speculate that this peculiar dynamics should be related with the motion of a particle in a conical geometry under the action of a potential $U(r)$, or from the perspective of gravity, with the dynamics in a global monopole spacetime [80]. In fact, generalizations of $\mathrm{SU}(2)$ systems with $D(2,1 ; \alpha)$ superconformal mechanics in Einstein-Maxwell background were studied recently in [81]. It would be very interesting to generalize the system with harmonic trap that we considered for the case of $D(2,1 ; \alpha)$ superconformal mechanics and to look for its relation with the systems from [81] in the light of the conformal bridge transformation. In another but somehow related direction, it could be interesting to study this system and its hidden symmetries from the perspective of Eisenhart-Duval lift [82] and Killing-Yano tensors [1].

\footnotetext{
${ }^{3}$ For earlier discussion of the quantum mechanical and classical aspects of such a universality see [14, 78, 79]. We thank A. Nersessian for drawing our attention to these works.
} 
The similarities in the dynamics are revealed not only at the classical level, but also in the quantum theory. In particular, the Hamiltonian operator (3.2) has the form of a threedimensional harmonic oscillator Hamiltonian with a modified angular momentum, which takes values $j=|\nu|+k$ with $k=0,1, \ldots$. Also, degeneracy of the spectrum, related with the ratio of the classical radial and angular periods, can be explained in terms of the hidden integrals of motion as we did in section 3.1. Earlier results on the quantum analogy was obtained for this system and for the Kepler potential in [65] in the case of integer values of $\nu$.

On the other hand, though the systems of the form (5.1) with $U=0$ and $U=\frac{1}{2} m \omega^{2} r^{2}$ classically and quantum mechanically are essentially different since their Hamiltonians are generators of conformal $\mathfrak{s l}(2, \mathbb{R})$ symmetry of non-compact and compact topological nature, respectively, they correspond to two different forms of dynamics governed by conformal symmetry in the sense of Dirac [33, 71]. This fact allowed us to relate them at the quantum level (that also can be done classically) by applying the conformal bridge transformation [34], as we did this in section 3.2. Symmetry generators of one of these systems, including those of hidden symmetry, are mapped into symmetries of the other system. This transformation also allowed us to obtain the coherent states for the system we studied.

In the second part, similarly to the construction of the Dirac oscillator [57], we introduce additional spin degrees of freedom at the quantum level and adding a spin-orbit coupling term $\pm \omega^{\prime} \boldsymbol{J} \cdot \boldsymbol{\sigma}$. The constant value $\omega^{\prime}=\omega$ is very special as then the spectrum is divided in two subsets. The eigenvalues in one subset do not depend on the quantum angular momentum number $j$ and hence are infinitely degenerate. In the other subset each energy level has finite degeneracy defined by the constant $\nu=e g$ which can only take integer and half-integer values. Using the hidden symmetries of the scalar system, as well as its conformal Newton-Hooke symmetry, we construct independent pairs of non-local ladder operators acting within both subspaces, one with infinite and one with finite degeneracy of energy levels. Here we do not compute commutators of these objects and the question on the symmetry algebra of the system remains unanswered.

The system with spin degrees of freedom gives rise to an $\mathcal{N}=2$ supersymmetric system characterized by the $\mathfrak{o} \mathfrak{s p}(2 \mid 2)$ superconformal symmetry. Applying two different dimensional reduction schemes to the obtained superconformal system produces in one case the one-dimensional superconformal extension of the AFF model with harmonic trap in the phase of the unbroken $\mathcal{N}=2$ Poincaré supersymmetry, while in the other case gives us the same system but in the spontaneously broken phase [19, 20, 71, 73]. In this context, it would be interesting to look for three-dimensional generalizations of the one-dimensional rationally deformed superconformal systems constructed recently in [19, 20, 71] by using dual Darboux transformations.

Hermitian supercharges of three-dimensional supersymmetric quantum mechanics can be related with $(3+1)$-dimensional Dirac operators in Euclidean space by setting $\partial_{t} \rightarrow$ 0 , and adding a gauge field connection. It is known that for self-dual or anti-self-dual electromagnetic fields an extended $\mathcal{N}=4$ supersymmetry can be obtained [39]. In the present case, the Hermitian combination $\mathcal{Q}^{+}+\mathcal{Q}^{-}$, where $\mathcal{Q}^{ \pm}$are given in (4.42), can be 
re-written in terms of Euclidean Dirac matrices

$$
\gamma^{i}=\left(\begin{array}{cc}
0 & -i \sigma_{i} \\
i \sigma_{i} & 0
\end{array}\right), \quad \gamma^{0}=\left(\begin{array}{ll}
0 & 1 \\
1 & 0
\end{array}\right)
$$

in the following form:

$$
\mathcal{Q}_{0}=-\sqrt{2}\left(\mathcal{Q}^{+}+\mathcal{Q}^{-}\right)=\gamma^{i}\left(p_{i}-e \mathscr{A}_{i}\right)+e \gamma^{0} \mathscr{A}_{0},
$$

where

$$
\mathscr{A}_{0}=\frac{g}{r}, \quad \mathscr{A}_{i}=A_{i}-i \frac{\omega}{e} \gamma^{5} r_{i},
$$

with $\gamma^{5}=\Gamma$ is our grading operator in section 4.2. Then the operator (5.4) can be viewed as a parity breaking Euclidean Dirac operator with components of the gauge potential satisfying the relations $-\partial_{i} \mathscr{A}_{0}=\epsilon_{i j k} \partial_{j} \mathscr{A}_{k}=g r_{i} / r^{3}$. Hence we are dealing with a new type of parity breaking dyon background. Actually, the $\gamma^{5}$ terms do not allow for an $\mathcal{N}=4$ supersymmetric extension and we only have $\mathcal{N}=2$ supersymmetry, with the second supercharge given by $i \sqrt{2}\left(\mathcal{Q}^{+}-\mathcal{Q}^{-}\right)=i \gamma^{5} \mathcal{Q}_{0}$. It is interesting to relate a paritybreaking Dirac operator with a supersymmetric quantum mechanics. In this context it is not clear whether a (pseudo)classical supersymmetric system exists whose quantization would produce our three-dimensional $\mathfrak{s l}(2, \mathbb{R})$ superconformal system, or we have here a kind of a classical anomaly [83].

To further interpret the three-dimensional supersymmetric system, one can study limiting cases of the coupling constant. In particular, in the limit $\nu \rightarrow 0$ we recover the non-relativistic limit of the Dirac oscillator considered in [56-60], and the supersymmetry (4.48)-(4.52) remains intact. On the other hand, in the limit, $\omega \rightarrow 0$, both Hamiltonians in (4.39) take the form

$$
\mathcal{H}_{\text {dyon }}=\frac{1}{2}\left(\pi^{2}+\frac{\nu^{2}}{r^{2}}-\frac{4 \nu}{r^{3}} \mathcal{S}_{i}^{-} \cdot r\right), \quad \mathcal{S}^{-}=\frac{1}{2}(1-\Gamma) \mathcal{S},
$$

where $\mathcal{S}=\mathbb{I} \otimes \frac{1}{2} \boldsymbol{\sigma}$ denotes the vector spin operator. This is just a Pauli type Hamiltonian for a charged spin-1/2 particle in a field of a self-dual dyon [32]. In the same limiting case the operator (5.4) is a Dirac type Hamiltonian which is identified as a supercharge related to (5.6). As we have emphasized earlier, this system has extended $\mathcal{N}=4$ supersymmetry. However, taking the limit $\omega \rightarrow 0$ in our system (and following the approach in [73]) we cannot reconstruct the other three supercharges and one may suspect that something is still missing in our construction. One possible way to answer this question is to try to perform a supersymmetric extension of the conformal bridge [34] and to apply this to the system (5.6).

Finally, another interesting question related to the Killing-Yano tensor problem mentioned above is the possible existence of an additional, hidden non-linear supersymmetry in the system studied by us in section 4.2. Such a possibility is suggested by the presence of such symmetries in the system of a spin-1/2 particle in a self-dual dyon background [32], in superconformal mechanics at special values of the boson-fermion coupling constant [84, 85], in the system of a scalar particle investigated by us in section 2.3 , and the nonlinear exotic supersymmetry seen in the systems of spinning particles in backgrounds characterized by the presence of Killing-Yano tensors [7, 25-28]. 


\section{Acknowledgments}

The work was partially supported by the CONICYT scholarship 21170053 (LI), FONDECYT Project 1190842 and DICYT, USACH (MSP), and the Project U.S.A. 1899 (MSP and LI). LI and MSP also thank FSU for hospitality during various stages of this work.

\section{A The monopole harmonics}

The monopole vector gauge potential possesses a singularity often called Dirac string, because of which one may split the domain of definition of this field in two parts (charts) related by a gauge transformation. The continuity conditions for the wave function in the transition region imply the remarkable result that $\nu=e g$ can take only integer and half integer values at the quantum level. Here we obtain an explicit expression for monopole harmonics, and for this purpose it is enough to work in the fixed gauge

$$
\boldsymbol{A}=\frac{g z}{r\left(x^{2}+y^{2}\right)}(y \hat{x}-x \hat{y})=-\frac{g}{r} \cot (\theta) \hat{\varphi}, \quad \hat{\varphi}=(-\sin \varphi, \cos \varphi) .
$$

To see how the monopole harmonics change under the corresponding gauge transformation, see refs. [67, 68]. With this choice, the spherical components of the Poincaré vector $\boldsymbol{J}$ are given by

$$
J_{ \pm}=e^{ \pm i \varphi}\left(i \cot \theta \frac{\partial}{\partial \varphi} \pm \frac{\partial}{\partial \theta}-\frac{\nu}{\sin \theta}\right), \quad J_{3}=-i \frac{\partial}{\partial \varphi} .
$$

These operators can be obtained as a "reduction" of the $\mathfrak{s o}(4)=\mathfrak{s o}(3) \oplus \mathfrak{s o}(3)$ symmetry

$$
\left[\mathscr{J}_{i}, \mathscr{J}_{j}\right]=i \epsilon_{i j k} \mathscr{J}_{k}, \quad\left[\mathscr{K}_{a}, \mathscr{K}_{b}\right]=i \epsilon_{a b c} \mathscr{K}_{c}, \quad\left[\mathscr{J}_{i}, \mathscr{K}_{a}\right]=0
$$

of the spinning top. Consider the following realization of this algebra [86],

$$
\begin{array}{ll}
\mathscr{J}_{ \pm}=\mathscr{J}_{1} \pm i \mathscr{J}_{2}=e^{ \pm i \varphi}\left(i \cot \theta \frac{\partial}{\partial \varphi} \pm \frac{\partial}{\partial \theta}-\frac{i}{\sin \theta} \frac{\partial}{\partial \psi}\right), & \mathscr{J}_{3}=-i \frac{\partial}{\partial \varphi}, \\
\mathscr{K}_{ \pm}=\mathscr{K}_{1} \pm i \mathscr{K}_{2}=e^{\mp i \psi}\left(i \cot \theta \frac{\partial}{\partial \psi} \mp \frac{\partial}{\partial \theta}-\frac{i}{\sin \theta} \frac{\partial}{\partial \varphi}\right), & \mathscr{K}_{3}=i \frac{\partial}{\partial \psi}
\end{array}
$$

Here, $\theta, \varphi$ and $\psi$ are the Euler angles, $0 \leq \varphi, \psi<2 \pi, 0 \leq \theta<\pi$, and $\mathscr{J}_{i} \mathscr{J}_{i}=\mathscr{K}_{a} \mathscr{K}_{a}=$ $\mathscr{J}^{2}$. The common eigenstates of $\mathscr{J}^{2}, \mathscr{J}_{3}$ and $\mathscr{K}_{3}$ satisfying relations

$$
\begin{array}{ll}
\mathscr{J}^{2} D_{j, m, m^{\prime}}=j(j+1) D_{j, m, m^{\prime}}, \quad \mathscr{J}_{ \pm} D_{j, m, m^{\prime}}=\sqrt{(j \mp m)(j \pm m+1)} D_{j, m \pm 1, m^{\prime}}, \\
\mathscr{K}_{ \pm} D_{j, m, m^{\prime}}=\sqrt{\left(j \mp m^{\prime}\right)\left(j \pm m^{\prime}+1\right)} D_{j, m, m^{\prime} \pm 1}, \\
\mathscr{J}_{3} D_{j, m, m^{\prime}}=m D_{j, m, m^{\prime}}, & \mathscr{K}_{3} D_{j, m, m^{\prime}}=m^{\prime} D_{j, m, m^{\prime}},
\end{array}
$$

are given by the generalized spherical functions

$$
D_{j, m, m^{\prime}}(\varphi, \theta, \psi)=e^{i\left(m \varphi+m^{\prime} \psi\right)} P_{j, m, m^{\prime}}(\cos \theta),
$$


where

$$
\begin{aligned}
P_{j, m, m^{\prime}}(u) & =\mathcal{N}_{j, m, m^{\prime}}(1-u)^{-\frac{m-m^{\prime}}{2}}(1+u)^{-\frac{m+m^{\prime}}{2}}\left(\frac{d}{d u}\right)^{j-m}(1-u)^{j-m^{\prime}}(1+u)^{j+m^{\prime}}, \\
\mathcal{N}_{j, m, m^{\prime}} & =\frac{(-1)^{j-m^{\prime}}}{2^{j}\left(j-m^{\prime}\right) !} \sqrt{\frac{(j-m) !\left(j+m^{\prime}\right) !}{(j+m) !\left(j-m^{\prime}\right) !}}, \quad m, m^{\prime}=j, j-1, \ldots,-j . \\
j & =0,1 / 2,1,3 / 2, \ldots, \quad \text { A. } .0)
\end{aligned}
$$

The necessary reduction is achieved by imposing the condition

$$
\left(\mathscr{K}_{3}-\nu\right) \Psi(\theta, \varphi, \psi)=0
$$

on a wave function $\Psi(\theta, \varphi, \psi)$ being a linear combination of the states $D_{j, m, m^{\prime}}(\varphi, \theta, \psi)$. This equation has a nontrivial solution only when a constant parameter $\nu$ takes some integer or half-integer value that corresponds to the Dirac quantization condition for $\nu=e g$. Fixing integer or half-integer value for $\nu$, a general solution of (A.11) is a linear combination of the states $D_{j, m, \nu}$, with $j=|\nu|,|\nu|+1, \ldots, m=-j,-j+1, \ldots, j$, and therefore the monopole harmonics are given by

$$
\mathcal{Y}_{j}^{j_{3}}(\theta, \phi ; \nu)=e^{-i \nu \psi} D_{j, j_{3}, \nu}(\theta, \phi, \psi)
$$

\section{B The derivation of $A_{n, j, m}$ and $B_{n, j, m}$}

To clarify the action of operators $\boldsymbol{a}^{ \pm}$it is convenient to introduce notation

$$
\eta_{0}=a_{3}, \quad \eta_{ \pm}=a_{1} \pm i a_{2}
$$

Then

$$
\begin{array}{rlrl}
{\left[H, \eta_{q}\right]} & =-\omega \eta_{q}, & {\left[J_{3}, \eta_{0}\right]} & =\left[J_{ \pm}, \eta_{ \pm}\right]=0, \quad\left[J_{3}, \eta_{ \pm}\right]= \pm \eta_{ \pm}, \quad q=0, \pm \\
{\left[J_{ \pm}, \eta_{0}\right]} & =\mp \eta_{ \pm}, \quad\left[J_{ \pm}, \eta_{\mp}\right]= \pm 2 \eta_{0} .
\end{array}
$$

From equations (B.2) one concludes that the action of $\eta_{q}$ and $\eta_{q}^{\dagger}$ has to be of the form

$$
\begin{aligned}
& \eta_{q}|n, j, m\rangle=A_{n, j, m}^{(q)}|n, j-1, m+q\rangle+B_{n, j, m}^{(q)}|n-1, j+1, m+q\rangle, \\
& \eta_{q}^{\dagger}|n, j, m\rangle=A_{n, j+1, m-q}^{(-q) *}|n, j+1, m-q\rangle+B_{n+1, j-1, m-q}^{(-q) *}|n+1, j-1, m-q\rangle .
\end{aligned}
$$

The first equation in (B.3) means that $A_{n, j, m}^{( \pm)}$and $B_{n, j, m}^{( \pm)}$are related with $A_{n, j, m}^{(0)} \equiv A_{n, j, m}$ and $B_{n, j, m}^{( \pm)} \equiv B_{n, j, m}$ by means of the algebraic expressions

$$
\begin{aligned}
& A_{n, j, m}^{( \pm)}=\mp \sqrt{(j \mp m-1)(j \pm m)} A_{n, j, m} \pm \sqrt{(j \mp m)(j \pm m+1)} A_{n, j, m \pm 1}, \\
& B_{n, j, m}^{( \pm)}=\mp \sqrt{(j \mp m+1)(j \pm m+2)} B_{n, j, m} \pm \sqrt{(j \mp m)(j \pm m+1)} B_{n, j, m \pm 1},
\end{aligned}
$$


and using the second equation in (B.3) we derive the recurrence relations

$$
\begin{aligned}
\frac{2\left(2 j+j^{2}-m^{2}\right) B_{n, j, m}}{\sqrt{(j+1)^{2}-m^{2}}=} & \sqrt{(j-m+2)(j+m+1)} B_{n, j, m-1} \\
& +\sqrt{(j+m+2)(j-m)} B_{n, j, m+1} \\
2\left(j^{2}-m^{2}-1\right) A_{n, j, m}= & {\left[j^{2}-m^{2}\right]^{-\frac{1}{2}}\left(\sqrt{j^{2}-(m-1)^{2}} A_{n, j, m-1}\right.} \\
& \left.+\sqrt{(j+1)^{2}-m^{2}} A_{n, j, m+1}\right),
\end{aligned}
$$

the solutions of which are

$$
A_{n, j, m}=\sqrt{(j+m)(j-m)} a_{n, j}, \quad B_{n, j, m}=\sqrt{(j+m+1)(j-m+1)} b_{n, j} .
$$

To determine coefficients $a_{n, j}$ and $b_{n, j}$ we use the relations

$$
\left[\eta_{0}, \eta_{ \pm 1}\right]=\mp \mathcal{C} J_{ \pm} \quad\left[\eta_{0}, \eta_{0}^{\dagger}\right]=\omega\left(2 \boldsymbol{J}^{2}-J_{3}^{2}+1-\nu^{2}\right)
$$

which produce the equations

$$
\begin{aligned}
(2 j+3) b_{n, j} a_{n-1, j+1}-(2 j-1) b_{n, j-1, m} a_{n, j}= & \omega \sqrt{2 n(2 n+2 j+1)}, \\
\omega\left(2 j(j+1)+1-\nu^{2}-m^{2}\right)= & \left.(2 j+1)\left(a_{n, j+1}^{2}-b_{n, j}^{2}\right)+\quad \text { (B. } 10\right) \\
& +\left(m^{2}-j^{2}\right)\left(a_{n, j}^{2}-b_{n+1, j-1}^{2}+b_{n, j}^{2}-a_{n, j+1}^{2}\right) .
\end{aligned}
$$

As $b_{n, j}$ and $a_{n, j}$ do not depend on $m$, the last equation implies the identity $a_{n, j}^{2}-b_{n+1, j-1}^{2}+$ $b_{n, j}^{2}-a_{n, j+1}^{2}=-\omega$. On the other hand, from equation (B.4) with $q=0$ we conclude that the constant $b_{0, j}$ should vanish, contrary to the constant $a_{0, j} \neq 0$. Using this and the first equation in (B.10) we have

$$
a_{n, j}=\tilde{a}_{n, j} \sqrt{\frac{\omega(j+\nu)(j-\nu)}{(2 j-1)}}, \quad \tilde{a}_{0, j+1}=1 .
$$

Inserting this result and the anzatz

$$
b_{n, j}=\tilde{b}_{j} \sqrt{\frac{\omega 2 n(j+\nu+1)(j-\nu+1)}{(2 j+1)}},
$$

into equations eq. (B.10) we finally obtain the system of equations

$$
(2 j+3) \tilde{b}_{j} \tilde{a}_{n-1, j+1}=\sqrt{2 n+2 j+1}, \quad 2 n \tilde{b}_{j}^{2}-\tilde{a}_{n, j+1}^{2}=-1
$$

which has the solutions $\tilde{b}_{j}^{2}=(2 j+3)$ and $\tilde{a}_{n, j}^{2}=\left(\frac{2 n+2 j+1}{2 j+1}\right)$. Collecting our results we end up with (3.18). 


\section{Generalized Laguerre polynomials}

When acting with the first order operators $\Theta, \Xi$ and their adjoint on the eigenspinors $\left|n, k, k_{3}, \pm\right\rangle$ and $\left.\| n, k, k_{3}, \pm\right\rangle$, the following functional relations for the generalized Laguerre polynomials are useful:

$$
\begin{aligned}
y \frac{d}{d y} L_{n}^{\alpha}(y)-y L_{n}^{\alpha}(y)+\alpha L_{n}^{\alpha} & =(n+1) L_{n+1}^{\alpha-1}, \\
\frac{d}{d y} L_{n}^{\alpha}(y)-L_{n}^{\alpha}(y) & =-L_{n}^{\alpha+1}(y), \\
\frac{d}{d y} L_{n}^{\alpha}(y) & =-L_{n-1}^{\alpha+1}(y), \\
y \frac{d}{d y} L_{n}^{\alpha}(y)+\alpha L_{n}^{\alpha}(y) & =(n+\alpha) L_{n}^{\alpha-1}(y) .
\end{aligned}
$$

\section{Commutators $[\mathcal{R}, \mathcal{Q}]$ and $\left[\mathcal{R}, \mathcal{W}^{\dagger}\right]$}

To compute these commutators, we first observe that $\boldsymbol{b}$ and $\boldsymbol{b}^{\dagger}$ are vector operators with respect to $\boldsymbol{J}$, that means

$$
\boldsymbol{J} \cdot \boldsymbol{b}=\boldsymbol{b} \cdot \boldsymbol{J}, \quad \boldsymbol{J} \times \boldsymbol{b}+\boldsymbol{b} \times \boldsymbol{J}=2 i \boldsymbol{b} .
$$

Next we use the equality $\boldsymbol{\pi} \times \boldsymbol{\pi}=i \frac{\nu}{r^{2}} \boldsymbol{n}$ which implies

$$
\boldsymbol{J} \cdot \boldsymbol{b}=\frac{1}{\sqrt{2} r}\left(i \nu-\nu \boldsymbol{r} \cdot \boldsymbol{\pi}+i \omega \nu r^{2}\right), \quad \boldsymbol{J} \cdot \boldsymbol{b}^{\dagger}=\frac{1}{\sqrt{2} r}\left(i \nu-\nu \boldsymbol{r} \cdot \boldsymbol{\pi}-i \omega \nu r^{2}\right) .
$$

With these identities and representations of $\Theta$ and $\Xi$ in (4.25) and (4.30), one can easily compute the commutators

$$
\begin{aligned}
{[\mathcal{R}, \mathcal{Q}] } & =\left(\begin{array}{l}
0\left(\boldsymbol{J} \cdot \boldsymbol{\sigma}+\frac{3}{2}\right) \Theta+\Theta\left(\boldsymbol{J} \cdot \boldsymbol{\sigma}+\frac{3}{2}+2 \nu \sigma_{r}\right) \\
0 \\
0
\end{array}\right)=\mathcal{Q}, \\
{\left[\mathcal{R}_{\nu}, \mathcal{W}^{\dagger}\right] } & =\left(\begin{array}{l}
0\left(\boldsymbol{J} \cdot \boldsymbol{\sigma}+\frac{3}{2}\right) \Xi+\Xi\left(\boldsymbol{J} \cdot \boldsymbol{\sigma}+\frac{3}{2}+2 \nu \sigma_{r}\right) \\
0
\end{array}\right)=\mathcal{W}^{\dagger} .
\end{aligned}
$$

Open Access. This article is distributed under the terms of the Creative Commons Attribution License (CC-BY 4.0), which permits any use, distribution and reproduction in any medium, provided the original author(s) and source are credited.

\section{References}

[1] M. Cariglia, Hidden symmetries of dynamics in classical and quantum physics, Rev. Mod. Phys. 86 (2014) 1283 [arXiv:1411.1262] [INSPIRE].

[2] O. Evnin and R. Nivesvivat, Hidden symmetries of the Higgs oscillator and the conformal algebra, J. Phys. A 50 (2017) 015202 [arXiv: 1604.00521] [INSPIRE]. 
[3] O. Evnin and C. Krishnan, A hidden symmetry of AdS resonances, Phys. Rev. D 91 (2015) 126010 [arXiv: 1502.03749] [INSPIRE].

[4] O. Evnin and R. Nivesvivat, AdS perturbations, isometries, selection rules and the Higgs oscillator, JHEP 01 (2016) 151 [arXiv:1512.00349] [INSPIRE].

[5] S.P. Novikov, S.V. Manakov, L.P. Pitaevskii and V.E. Zakharov, Theory of solitons, Plenum, New York, NY, U.S.A. (1984).

[6] F. Correa, O. Lechtenfeld and M. Plyushchay, Nonlinear supersymmetry in the quantum Calogero model, JHEP 04 (2014) 151 [arXiv:1312.5749] [INSPIRE].

[7] V. Frolov, P. Krtous and D. Kubiznak, Black holes, hidden symmetries and complete integrability, Living Rev. Rel. 20 (2017) 6 [arXiv:1705.05482] [INSPIRE].

[8] A. Kirchberg, J.D. Lange, P.A.G. Pisani and A. Wipf, Algebraic solution of the supersymmetric hydrogen atom in d-dimensions, Annals Phys. 303 (2003) 359 [hep-th/0208228] [INSPIRE].

[9] E. Ivanov, S. Krivonos and O. Lechtenfeld, New variant of $N=4$ superconformal mechanics, JHEP 03 (2003) 014 [hep-th/0212303] [INSPIRE].

[10] F. Correa, V. Jakubsky, L.-M. Nieto and M.S. Plyushchay, Self-isospectrality, special supersymmetry and their effect on the band structure, Phys. Rev. Lett. 101 (2008) 030403 [arXiv:0801.1671] [INSPIRE].

[11] M.S. Plyushchay, Nonlinear supersymmetry as a hidden symmetry, in Integrability, supersymmetry and coherent states, S.. Kuru, J. Negro and L.M. Nieto eds., CRM Series in Mathematical Physics, Springer, Cham, Switzerland (2019), pg. 163 [arXiv:1811.11942] [INSPIRE].

[12] D.M. Fradkin, Three-dimensional isotropic harmonic oscillator and $\mathrm{SU}_{3}$, Amer. J. Phys. 33 (1965) 207.

[13] W. Pauli, Über das Wasserstoffspektrum vom Standpunkt der neuen Quantenmechanik (in German), Z. Phys. 36 (1926) 336.

[14] D. Zwanziger, Exactly soluble nonrelativistic model of particles with both electric and magnetic charges, Phys. Rev. 176 (1968) 1480 [INSPIRE].

[15] A. Arancibia and M.S. Plyushchay, Chiral asymmetry in propagation of soliton defects in crystalline backgrounds, Phys. Rev. D 92 (2015) 105009 [arXiv:1507.07060] [INSPIRE].

[16] J.F. Cariñena and M.S. Plyushchay, ABC of ladder operators for rationally extended quantum harmonic oscillator systems, J. Phys. A 50 (2017) 275202 [arXiv:1701.08657] [INSPIRE].

[17] J. Mateos Guilarte and M.S. Plyushchay, Perfectly invisible PT-symmetric zero-gap systems, conformal field theoretical kinks and exotic nonlinear supersymmetry, JHEP 12 (2017) 061 [arXiv: 1710.00356] [INSPIRE].

[18] J. Mateos Guilarte and M.S. Plyushchay, Nonlinear symmetries of perfectly invisible PT-regularized conformal and superconformal mechanics systems, JHEP 01 (2019) 194 [arXiv: 1806.08740] [INSPIRE].

[19] J.F. Cariñena, L. Inzunza and M.S. Plyushchay, Rational deformations of conformal mechanics, Phys. Rev. D 98 (2018) 026017 [arXiv: 1707.07357] [INSPIRE].

[20] L. Inzunza and M.S. Plyushchay, Hidden symmetries of rationally deformed superconformal mechanics, Phys. Rev. D 99 (2019) 025001 [arXiv: 1809.08527] [INSPIRE]. 
[21] J. de Boer, F. Harmsze and T. Tjin, Nonlinear finite $W$ symmetries and applications in elementary systems, Phys. Rept. 272 (1996) 139 [hep-th/9503161] [INSPIRE].

[22] E. D'Hoker and L. Vinet, Supersymmetry of the Pauli equation in the presence of a magnetic monopole, Phys. Lett. B 137 (1984) 72 [INSPIRE].

[23] E. D'Hoker and L. Vinet, Dynamical supersymmetry of the magnetic monopole and the $1 / r^{2}$ potential, Commun. Math. Phys. 97 (1985) 391 [INSPIRE].

[24] F. De Jonghe, A.J. Macfarlane, K. Peeters and J.W. van Holten, New supersymmetry of the monopole, Phys. Lett. B 359 (1995) 114 [hep-th/9507046] [INSPIRE].

[25] G.W. Gibbons, R.H. Rietdijk and J.W. van Holten, SUSY in the sky, Nucl. Phys. B 404 (1993) 42 [hep-th/9303112] [INSPIRE].

[26] M. Tanimoto, The role of Killing-Yano tensors in supersymmetric mechanics on a curved manifold, Nucl. Phys. B 442 (1995) 549 [gr-qc/9501006] [INSPIRE].

[27] M. Cariglia, Quantum mechanics of Yano tensors: Dirac equation in curved spacetime, Class. Quant. Grav. 21 (2004) 1051 [hep-th/0305153] [INSPIRE].

[28] M.S. Plyushchay, On the nature of fermion monopole supersymmetry, Phys. Lett. B 485 (2000) 187 [hep-th/0005122] [inSPIRE].

[29] P. Goddard and D.I. Olive, New developments in the theory of magnetic monopoles, Rept. Prog. Phys. 41 (1978) 1357 [inSPIRE].

[30] M.S. Plyushchay, Monopole Chern-Simons term: charge monopole system as a particle with spin, Nucl. Phys. B 589 (2000) 413 [hep-th/0004032] [INSPIRE].

[31] M.S. Plyushchay, Free conical dynamics: charge-monopole as a particle with spin, anyon and nonlinear fermion-monopole supersymmetry, Nucl. Phys. Proc. Suppl. 102 (2001) 248 [hep-th/0103040] [INSPIRE].

[32] M.S. Plyushchay and A. Wipf, Particle in a self-dual dyon background: hidden free nature and exotic superconformal symmetry, Phys. Rev. D 89 (2014) 045017 [arXiv:1311.2195] [INSPIRE].

[33] P.A.M. Dirac, Forms of relativistic dynamics, Rev. Mod. Phys. 21 (1949) 392 [inSPIRE].

[34] L. Inzunza, M.S. Plyushchay and A. Wipf, Conformal bridge between freedom and confinement, arXiv:1912.11752 [INSPIRE].

[35] E. Witten, Dynamical breaking of supersymmetry, Nucl. Phys. B 188 (1981) 513 [InSPIRE].

[36] E. Witten, Constraints on supersymmetry breaking, Nucl. Phys. B 202 (1982) 253 [INSPIRE].

[37] V.B. Matveev and M.A. Salle, Darboux transformations and solitons, Springer, Berlin, Germany (1991).

[38] F. Cooper, A. Khare and U. Sukhatme, Supersymmetry and quantum mechanics, Phys. Rept. 251 (1995) 267 [hep-th/9405029] [INSPIRE].

[39] A. Kirchberg, J.D. Lange and A. Wipf, Extended supersymmetries and the Dirac operator, Annals Phys. 315 (2005) 467 [hep-th/0401134] [InSPIRE].

[40] S. Bellucci, S. Krivonos and A. Nersessian, $N=8$ supersymmetric mechanics on special Kähler manifolds, Phys. Lett. B 605 (2005) 181 [hep-th/0410029] [InSPIRE]. 
[41] S. Bellucci, A. Nersessian and A. Yeranyan, Hamiltonian reduction and supersymmetric mechanics with Dirac monopole, Phys. Rev. D 74 (2006) 065022 [hep-th/0606152] [INSPIRE].

[42] N. Kozyrev, S. Krivonos, O. Lechtenfeld, A. Nersessian and A. Sutulin, Curved Witten-Dijkgraaf-Verlinde-Verlinde equation and $N=4$ mechanics, Phys. Rev. D 96 (2017) 101702 [arXiv: 1710.00884] [INSPIRE].

[43] N. Kozyrev, S. Krivonos, O. Lechtenfeld, A. Nersessian and A. Sutulin, $N=4$ supersymmetric mechanics on curved spaces, Phys. Rev. D 97 (2018) 085015 [arXiv: 1711.08734] [INSPIRE].

[44] V. de Alfaro, S. Fubini and G. Furlan, Conformal invariance in quantum mechanics, Nuovo Cim. A 34 (1976) 569 [INSPIRE].

[45] S. Fubini and E. Rabinovici, Superconformal quantum mechanics, Nucl. Phys. B 245 (1984) 17 [INSPIRE].

[46] S. Fedoruk, E. Ivanov and O. Lechtenfeld, Superconformal mechanics, J. Phys. A 45 (2012) 173001 [arXiv: 1112.1947] [INSPIRE].

[47] R. Britto-Pacumio, J. Michelson, A. Strominger and A. Volovich, Lectures on superconformal quantum mechanics and multi-black hole moduli spaces, NATO Sci. Ser. C 556 (2000) 255 [hep-th/9911066] [INSPIRE].

[48] P. Claus, M. Derix, R. Kallosh, J. Kumar, P.K. Townsend and A. Van Proeyen, Black holes and superconformal mechanics, Phys. Rev. Lett. 81 (1998) 4553 [hep-th/9804177] [INSPIRE].

[49] J.A. de Azcárraga, J.M. Izquierdo, J.C. Pérez Bueno and P.K. Townsend, Superconformal mechanics and nonlinear realizations, Phys. Rev. D 59 (1999) 084015 [hep-th/9810230] [INSPIRE].

[50] G.W. Gibbons and P.K. Townsend, Black holes and Calogero models, Phys. Lett. B 454 (1999) 187 [hep-th/9812034] [INSPIRE].

[51] J. Michelson and A. Strominger, Superconformal multiblack hole quantum mechanics, JHEP 09 (1999) 005 [hep-th/9908044] [INSPIRE].

[52] C. Chamon, R. Jackiw, S.-Y. Pi and L. Santos, Conformal quantum mechanics as the CFT dual to $A d S_{2}$, Phys. Lett. B 701 (2011) 503 [arXiv:1106.0726] [INSPIRE].

[53] B. Pioline and A. Waldron, Quantum cosmology and conformal invariance, Phys. Rev. Lett. 90 (2003) 031302 [hep-th/0209044] [INSPIRE].

[54] J. Ben Achour and E.R. Livine, Cosmology as a CFT, JHEP 12 (2019) 031 [arXiv: 1909.13390] [INSPIRE].

[55] S.J. Brodsky, G.F. de Teramond, H.G. Dosch and J. Erlich, Light-front holographic QCD and emerging confinement, Phys. Rept. 584 (2015) 1 [arXiv:1407.8131] [INSPIRE].

[56] A.B. Balantekin, Accidental degeneracies and supersymmetric quantum mechanics, Annals Phys. 164 (1985) 277 [INSPIRE].

[57] M. Moshinsky and A. Szczepaniak, The Dirac oscillator, J. Phys. A 22 (1989) L817.

[58] J. Benitez, R.P. Martinez y Romero, H.N. Núñez-Yépez and A.L. Salas-Brito, Solution and hidden supersymmetry of a Dirac oscillator, Phys. Rev. Lett. 64 (1990) 1643 [InSPIRE].

[59] C. Quesne and M. Moshinsky, Symmetry Lie algebra of the Dirac oscillator, J. Phys. A 23 (1990) 2263. 
[60] C. Quesne, Supersymmetry and the Dirac oscillator, Int. J. Mod. Phys. A 6 (1991) 1567 [INSPIRE].

[61] U. Niederer, The maximal kinematical invariance group of the harmonic oscillator, Helv. Phys. Acta 46 (1973) 191 [inSPIRE].

[62] A. Galajinsky, Conformal mechanics in Newton-Hooke spacetime, Nucl. Phys. B 832 (2010) 586 [arXiv: 1002.2290] [INSPIRE].

[63] K. Andrzejewski, Conformal Newton-Hooke algebras, Niederer's transformation and Pais-Uhlenbeck oscillator, Phys. Lett. B 738 (2014) 405 [arXiv: 1409.3926] [INSPIRE].

[64] A. Galajinsky, Geometry of the isotropic oscillator driven by the conformal mode, Eur. Phys. J. C 78 (2018) 72 [arXiv: 1712.00742] [INSPIRE].

[65] S. Labelle, M. Mayrand and L. Vinet, Symmetries and degeneracies of a charged oscillator in the field of a magnetic monopole, J. Math. Phys. 32 (1991) 1516 [InSPIRE].

[66] H.V. Mcintosh and A. Cisneros, Degeneracy in the presence of a magnetic monopole, $J$. Math. Phys. 11 (1970) 896 [INSPIRE].

[67] T.T. Wu and C.N. Yang, Dirac monopole without strings: monopole harmonics, Nucl. Phys. B 107 (1976) 365 [INSPIRE].

[68] T.T. Wu and C.N. Yang, Some properties of monopole harmonics, Phys. Rev. D 16 (1977) 1018 [INSPIRE].

[69] G. Lochak, Wave equation for a magnetic monopole, Int. J. Theor. Phys. 24 (1985) 1019 [INSPIRE].

[70] M.S. Plyushchay, Quantization of the classical $\mathrm{SL}(2, R)$ system and representations of $\mathrm{SL}(2, R)$ group, J. Math. Phys. 34 (1993) 3954 [INSPIRE].

[71] L. Inzunza and M.S. Plyushchay, Klein four-group and Darboux duality in conformal mechanics, Phys. Rev. D 99 (2019) 125016 [arXiv: 1902.00538] [INSPIRE].

[72] A. Perelomov, Generalized coherent states and their applications, Springer-Verlag, Berlin, Germany (1986).

[73] L. Inzunza and M.S. Plyushchay, Hidden superconformal symmetry: where does it come from?, Phys. Rev. D 97 (2018) 045002 [arXiv:1711.00616] [inSPIRE].

[74] L.D. Landau and E.M. Lifshitz, Quantum mechanics, Course of Theoretical Physics, volume 3, Pergamon Press, Oxford, U.K. (1965).

[75] H. Falomir, P.A.G. Pisani and A. Wipf, Pole structure of the Hamiltonian zeta function for a singular potential, J. Phys. A 35 (2002) 5427 [math-ph/0112019] [INSPIRE].

[76] H. Falomir and P.A.G. Pisani, Self-adjoint extensions and SUSY breaking in supersymmetric quantum mechanics, J. Phys. A 38 (2005) 4665 [hep-th/0501083] [INSPIRE].

[77] K. Kirsten and P. Loya, Spectral functions for the Schrödinger operator on $R^{+}$with a singular potential, J. Math. Phys. 51 (2010) 053512.

[78] A. Nersessian, Elements of (super-)Hamiltonian formalism, Lect. Notes Phys. 698 (2006) 139 [hep-th/0506170] [INSPIRE].

[79] L. Mardoyan, A. Nersessian and A. Yeranyan, Relationship between quantum mechanics with and without monopoles, Phys. Lett. A 366 (2007) 30 [hep-th/0610301] [INSPIRE]. 
[80] M. Barriola and A. Vilenkin, Gravitational field of a global monopole, Phys. Rev. Lett. 63 (1989) 341 [INSPIRE].

[81] A. Galajinsky and O. Lechtenfeld, Spinning extensions of $D(2,1 ; \alpha)$ superconformal mechanics, JHEP 03 (2019) 069 [arXiv:1902.06851] [INSPIRE].

[82] M. Cariglia, A. Galajinsky, G.W. Gibbons and P.A. Horvathy, Cosmological aspects of the Eisenhart-Duval lift, Eur. Phys. J. C 78 (2018) 314 [arXiv:1802.03370] [InSPIRE].

[83] J. Gamboa and M. Plyushchay, Classical anomalies for spinning particles, Nucl. Phys. B 512 (1998) 485 [hep-th/9711170] [INSPIRE].

[84] C. Leiva and M.S. Plyushchay, Superconformal mechanics and nonlinear supersymmetry, JHEP 10 (2003) 069 [hep-th/0304257] [INSPIRE].

[85] A. Anabalon and M.S. Plyushchay, Interaction via reduction and nonlinear superconformal symmetry, Phys. Lett. B 572 (2003) 202 [hep-th/0306210] [INSPIRE].

[86] L.C. Biedenharn, J.D. Louck and P.A. Carruthers, Angular momentum in quantum physics: theory and application, Oxford University Press, Oxford, U.K. (2009). 